\title{
Vortex clusters and multiquanta flux lattices in thin films of anisotropic superconductors
}

\author{
A. V. Samokhvalov ${ }^{(1)}$, D. A. Savinov ${ }^{(1)}$, A. S. Mel'nikov ${ }^{(1)}$, A. I. Buzdin ${ }^{(2)}$ \\ (1) Institute for Physics of Microstructures, \\ Russian Academy of Sciences, \\ 603950 Nizhny Novgorod, GSP-105, Russia \\ (2) Institut Universitaire de France and Universite Bordeaux I, France
}

\begin{abstract}
The distinctive features of equilibrium vortex structures in thin films of anisotropic superconductors in tilted magnetic fields are studied for the limits of moderate and strong anisotropy. The energetically favorable shape of isolated vortex lines is found in the framework of two particular models describing these limiting cases: London theory with an anisotropic mass tensor and London-type model for a stack of Josephson-decoupled superconducting layers. The increase of the field tilting is shown to result in qualitative changes in the vortex-vortex interaction potential: the balance between long-range attractive and repulsive forces occurs to be responsible for a formation of a minimum of the interaction potential vs the intervortex distance. This minimum appears to exist only for a certain restricted range of the vortex tilting angles which shrinks with the decrease of the system anisotropy parameter. Tilted vortices with such unusual interaction potential form clusters with the size depending on the field tilting angle and film thickness or/and can arrange into multiquanta flux lattice. The magnetic flux through the unit cells of the corresponding flux line lattices equals to an integer number $M$ of flux quanta. Thus, the increase in the field tilting should be accompanied by the series of the phase transitions between the vortex lattices with different $M$.
\end{abstract}

PACS numbers: 


\section{INTRODUCTION}

According to a standard picture of the mixed state in bulk type-II superconductors the Abrikosov vortices penetrating the homogeneous sample form a periodic arrangement called a flux lattice ${ }^{1}$. The magnetic flux through the unit cell of such flux line lattice equals to the flux quantum $\phi_{0}=\pi \hbar c / e$ : we have one vortex per unit cell. There are a few examples of rather exotic superconducting systems which may provide a possibility to observe a different vortex lattice periodicity, namely the structures with more than one vortices per unit cell. In particular, the phase transitions to such multiquanta flux lattices can occur, e.g., for superconductors with unconventional pairing 2,3 or 2D Fulde-Ferrell-Larkin-Ovchinnikov superconductors $\stackrel{4}{ }$.

The goal of this work is to suggest an alternative scenario of the phase transitions between the flux structures with different number of vortices per unit cell which can be realized in thin films of anisotropic superconductors. The underlying physical mechanism for this scenario arises from the interplay between the long range attraction and repulsion between

tilted vortex lines in thin films discussed recently in Ref. 5. The unusual attractive part of the vortex-vortex interaction potential is known to be a distinctive feature of anisotropic superconductors and the value of the attractive force is controlled by the tilting angle of the vortex line with respect to the anisotropy axis $\underline{\underline{6}} \underline{-\underline{8}}$. The origin of the long range intervortex repulsion in thin films has been analyzed in the pioneering work ${ }^{10}$ by Pearl in 1964. This repulsion force always overcomes the attraction at rather large distances because of the different power decay laws of these contributions. Note that, of course, the short range interaction between vortices is also repulsive. Finally, this balance between the repulsion and attraction can result in the formation of the nonmonotonic interaction potential $U(R)$ vs the intervortex distance $R$. Increasing the vortex tilting angle we first strengthen the attraction force between vortices and, thus, the minimum in the vortex interaction potential can appear only for rather large tilting angles when the attraction overcomes the Pearl's repulsion. This minimum shifts towards the larger intervortex distances with the further increase in the tilting angle and, finally, at rather large distances the attraction appears to be suppressed due to the exponential screening effect. As a consequence, the minimum in the interaction potential exists only for a certain restricted range of the vortex tilting angles which shrinks with the decrease of the system anisotropy parameter. The appearance of a 
minimum in the interaction potential points to the possibility to get a bound vortex pair (or even the clusters with higher vorticities) for a certain range of vortex tilting angles. For a flux line lattice such vortex-vortex interaction potential can cause an instability with respect to the unit cell doubling, i.e. the phase transition to the multiquanta vortex lattices.

In this paper we use two theoretical approaches to describe the peculiarities of the intervortex interaction and resulting formation of clusters and multiquanta lattices. One of them is a standard London model accounting for an anisotropic mass tensor which is adequate for the superconductors with moderate anisotropy. This approach assumes that the superconducting coherence length in all directions exceeds the distance between the atomic layers and obviously breaks down in the limit of strong anisotropy, i.e., for Josephson-coupled layered structures. In the latter case we choose to apply another phenomenological model, namely the so-called Lowrence-Doniach theory $\stackrel{11}{ }$. For rather small intervortex distances this theory can be simplified neglecting the effects of weak interlayer Josephson coupling. This approach of Josephson-decoupled superconducting layers is known to be useful in studies of the vortex-lattice structure at low fields 12,13 .

Considering thin film samples in tilted magnetic fields we do not restrict ourselves by the case of only straight vortex lines and study the problem of the energetically favorable vortex line shape in the presence of the inhomogeneous supercurrent screening the field component $\mathbf{H}_{\|}$parallel to the film plane. Previously this problem has been addressed in Ref. 14 for rather small deviations of the vortex line from the direction normal to the film plane. Such approximation is obviously valid only for the $\left|\mathbf{H}_{\|}\right|$values much smaller than the critical field $H_{c 1}^{(0)}$ of the penetration of vortices parallel to the film plane. For anisotropic London model this analysis of Ref. 14 has been previously generalized for the case of a strongly distorted vortex line (see Ref. 15). For the sake of completeness we present here the calculations of the shape of an isolated vortex line for arbitrary fields $\left|\mathbf{H}_{\|}\right|<H_{c 1}^{(0)}$ within both theoretical models describing the limits of strong and moderate anisotropy. As a next step, we calculate the vortex-vortex interaction potential for such strongly deformed vortex lines. Further analysis in the paper includes the calculations of energy of finite size vortex clusters as well as the energy of vortex lattices with different number of vortices per unit cell.

Experimentally the visualization of unconventional vortex arrangements could be carried out by a number of methods which provided convincing evidence for the existence 
of vortex chains in bulk anisotropic superconductors caused by the intervortex attraction phenomenon (such as the decoration technique in $\mathrm{YBa}_{2} \mathrm{Cu}_{3} \mathrm{O}_{7} \frac{16}{}$, scanning-tunneling microscopy in $\mathrm{NbSe}_{2} \frac{17}{}$, scanning Hall-probe $\frac{18}{}$ and Lorentz microscopy measurements in $\left.\mathrm{YBa}_{2} \mathrm{Cu}_{3} \mathrm{O}_{7} \frac{19,20}{2}\right)$.

The paper is organized as follows. In Sec. II we find the energetically favorable shape of an isolated vortex line. In Sec. III we calculate the vortex-vortex interaction potential and prove the existence of a potential minimum for a certain range of field tilting angles and parameters. The Sec. IV is devoted to the calculation of energy of vortex clusters. Finally, in Sec. $\mathrm{V}$ we present our analysis of the phase transition between the vortex lattices with one and two flux quanta per unit cell. The results are summarized in Sec. VI. Some of the calculation details are presented in the Appendices $\mathrm{A}$ and $\mathrm{B}$.

\section{ENERGETICALLY FAVORABLE SHAPE OF AN ISOLATED VORTEX LINE}

\section{A. Vortex line in a finite stack of thin superconducting layers}

We start our study of the distinctive features of equilibrium vortex structures in thin films of anisotropic superconductors with the consideration of the vortex line shape in the layered systems. Let us consider a finite stack of $N$ superconducting (SC) layers. Vortex line of an arbitrary shape pierces the film and can be viewed as a string of $2 \mathrm{D}$ pancake vortices: each of these pancakes is centered at the point $\mathbf{r}_{n}=x_{n} \mathbf{x}_{0}+y_{n} \mathbf{y}_{0}$ in the $n$-th layer. Within the model of the stack of Josephson-decoupled SC layers, pancakes can interact with each other only via magnetic fields. We denote the interlayer spacing as $s$ and consider each of the $N$ layers as a thin film with the thickness $d$ much less than the London penetration depth $\lambda$. General equation for the vector potential A distribution in such system reads

$$
\operatorname{rot} \operatorname{rot} \mathbf{A}=\frac{4 \pi}{c} \sum_{n, m=1}^{N} \mathbf{J}_{n}^{m}(\mathbf{r}) \delta\left(z-z_{n}\right),
$$

where $\Lambda=\lambda^{2} / d$ is the effective penetration depth in a superconducting film of a vanishing thickness $d$, each $n$-th SC layer coincides with the plane $z=z_{n}=n s(1 \leq n \leq N)$, the sheet current at the $n$-th layer created by the pancake at $m$-th layer takes the form

$$
\mathbf{J}_{n}^{m}(\mathbf{r})=\frac{c}{4 \pi \Lambda}\left[\mathbf{\Phi}\left(\mathbf{r}-\mathbf{r}_{m}\right) \delta_{n m}-\mathbf{A}^{m}\left(\mathbf{r}, z_{n}\right)\right]
$$


$\mathbf{A}^{m}(\mathbf{r}, z)$ is the vector potential induced by the only pancake vortex located in the $m$-th layer (Fig. 1). The vector $\mathbf{\Phi}(\mathbf{r})$ in the Eq. (2) is given by the expression

$$
\boldsymbol{\Phi}(\mathbf{r})=\frac{\Phi_{0}}{2 \pi} \frac{\left[\mathbf{z}_{0} \times \mathbf{r}\right]}{\mathbf{r}^{2}},
$$

and $\phi_{0}=\pi \hbar c / e$ is the flux quantum. For the layered system without Josephson coupling a general expression for the free energy can be written in the form:

$$
F=\frac{1}{8 \pi} \int d V\left[(\operatorname{rot} \mathbf{A})^{2}+\left(\frac{4 \pi}{c}\right)^{2} \Lambda \sum_{n} \mathbf{J}_{n}^{2}(\mathbf{r}) \delta\left(z-z_{n}\right)\right] .
$$

where the total vector potential $\mathbf{A}(\mathbf{r}, z)$ and the sheet current in the $n$-th layer $\mathbf{J}_{n}(\mathbf{r})$, produced by an arbitrary vortex line are the sum of the contributions induced by all $2 \mathrm{D}$ pancakes:

$$
\mathbf{A}(\mathbf{r}, z)=\sum_{m=1}^{N} \mathbf{A}^{m}(\mathbf{r}, z), \quad \mathbf{J}_{n}(\mathbf{r})=\sum_{m=1}^{N} \mathbf{J}_{n}^{m}(\mathbf{r}) .
$$

To find the magnetic vector potential $\mathbf{A}^{m}(\mathbf{r}, z)$ we adopt an approach similar to that in Refs. 21,22. Between the SC layers the vector potential $\mathbf{A}^{m}$ is described by the Laplace equation

$$
\triangle \mathrm{A}^{m}(\mathbf{r}, z)=0 .
$$

For the gauge $A_{z}^{m}=0$ the vector potential has only the in-plane components $\mathbf{A}^{m}=$ $\left(A_{x}^{m}, A_{y}^{m}\right)$, where

$$
\mathbf{A}^{m}(\mathbf{r}, z)=\frac{1}{(2 \pi)^{3}} \int d \mathbf{q} \mathrm{e}^{i \mathbf{q} \mathbf{r}} \mathbf{A}_{q}^{m} U^{m}(\mathbf{q}, z),
$$

and the function $U^{m}(\mathbf{q}, z)$ can be written as

$$
U^{m}(\mathbf{q}, z)=\left\{\begin{array}{c}
{\left[\alpha_{n}^{m} \sinh q\left(z_{n+1}-z\right)+\alpha_{n+1}^{m} \sinh q\left(z-z_{n}\right)\right] / \sinh (q s),} \\
z_{n}<z<z_{n+1}, n=1 \ldots N-1, \\
\alpha_{N}^{m} \exp \left(-q\left(z-z_{N}\right)\right), \quad z \geq z_{N}, \\
\alpha_{1}^{m} \exp \left(q\left(z-z_{1}\right)\right), \quad z \leq z_{1} .
\end{array}\right.
$$

Taking the Fourier transform of Eq. (2) we find:

$$
\mathbf{J}_{n}^{m}(\mathbf{q})=\frac{c}{4 \pi \Lambda}\left[\mathbf{\Phi}(\mathbf{q}) \mathrm{e}^{i \mathbf{q r}_{m}} \delta_{n m}-\mathbf{A}_{q}^{m} \alpha_{n}^{m}(\mathbf{q})\right],
$$


where

$$
\boldsymbol{\Phi}(\mathbf{q})=-i \phi_{0} \frac{\left[\mathbf{z}_{0} \times \mathbf{q}\right]}{q^{2}} .
$$

The sheet current density $\mathbf{J}_{n}^{m}$ results in the discontinuity of the in-plane component of the magnetic field $\mathbf{B}_{\|}^{m}$ across the $n$ layer:

$$
\frac{4 \pi}{c} \mathbf{J}_{n}^{m}=\mathbf{z}_{0} \times\left[\mathbf{B}_{\|}^{m}\left(\mathbf{r}, z_{n}+0\right)-\mathbf{B}_{\|}^{m}\left(\mathbf{r}, z_{n}-0\right)\right]=\mathbf{z}_{0} \times\left.\left[\mathbf{z}_{0} \times \frac{\partial \mathbf{A}^{m}}{\partial z}\right]\right|_{z_{n}-0} ^{z_{n}+0} .
$$

Substituting the expressions (6) , (7), (8) into above condition (10) we obtain the system of linear equations for the coefficients $\alpha_{n}^{m}$ :

$$
\begin{aligned}
& h(q) \alpha_{1}^{m}-\alpha_{2}^{m}=\delta_{1 m}, \\
- & \alpha_{n-1}^{m}+g(q) \alpha_{n}^{m}-\alpha_{n+1}^{m}=\delta_{n m}, \quad n=2 \ldots N-1, \\
- & \alpha_{N-1}^{m}+h(q) \alpha_{N}^{m}=\delta_{N m} .
\end{aligned}
$$

Here we introduce two new functions which depend on the wave number $q$ :

$$
g(q)=2 \cosh (q s)+\sinh (q s) / \Lambda q, \quad h(q)=\cosh (q s)+(1+1 / \Lambda q) \sinh (q s) .
$$

The solution of the linear system (111) and the Eqs. (6), (7) define the distribution of the vector potential $\mathbf{A}^{m}(\mathbf{r}, z)$ which is created by a single pancake vortex positioned in the $m$-th layer.

Without the in-plane external magnetic field $\mathbf{H}_{\|}$the relative displacement of the pancakes in the different layers is absent: $\mathbf{R}_{m k}=\mathbf{r}_{m}-\mathbf{r}_{k}=0$ (i.e. the pancakes form a vertical stack). A rather small magnetic field $\mathbf{H}_{\|}=H_{a} \mathbf{y}_{0}$ induces a screening Meissner current $\mathbf{J}_{n}^{M}=J_{n}^{M} \mathbf{x}_{0}$ in each $n$-th layer. Lorentz forces arising from these currents will move the pancakes from their initial positions. Taking into account the Eq. (10), we find the following system of linear equations describing the distribution of the magnetic field screened by the layered structure:

$$
\begin{aligned}
& \left(2+\frac{s}{\Lambda}\right) H_{1}-H_{2}=H_{a} \\
- & H_{n-1}+\left(2+\frac{s}{\Lambda}\right) H_{n}-H_{n+1}=0, \quad n=2 \ldots N-2, \\
- & H_{N-2}+\left(2+\frac{s}{\Lambda}\right) H_{N-1}=H_{a} .
\end{aligned}
$$


Here $\mathbf{H}_{n}=H_{n} \mathbf{y}_{0}$ is the magnetic field value between the $n$-th and $(n+1)$-th layers. The distribution of the Meissner screening currents in the layers takes the form:

$$
J_{1}^{M}=\frac{c}{4 \pi}\left(H_{1}-H_{a}\right), \quad J_{n}^{M}=\frac{c}{4 \pi}\left(H_{n}-H_{n-1}\right), n=2 \ldots N-2, \quad J_{N}^{M}=\frac{c}{4 \pi}\left(H_{a}-H_{N-1}\right) .
$$

The resulting Lorentz forces $\mathbf{F}_{n}^{M}$ acting on the pancakes can be written as follows:

$$
\mathbf{F}_{n}^{M}=\left(\phi_{0} / c\right)\left[\mathbf{J}_{n}^{M} \times \mathbf{z}_{0}\right]=\left(\phi_{0} / c\right) J_{n}^{M} \mathbf{y}_{0} .
$$

The interaction forces between the pancakes can be found using the expression (8) for the sheet current $\mathbf{J}_{k}^{m}$ generated by the pancake positioned in the $m$-th layer:

$$
\mathbf{F}_{k}^{m}=\left(\phi_{0} / c\right)\left[\mathbf{J}_{k}^{m} \times \mathbf{z}_{0}\right]=\frac{\phi_{0}^{2}}{8 \pi^{2} \Lambda \lambda_{a b}}\left\{\frac{1}{R_{m k}} \delta_{m k}-\int_{0}^{\infty} d q J_{1}\left(q R_{m k}\right) \frac{\alpha_{k}^{m}(q) g(q)}{Z(q)}\right\} \frac{\mathbf{R}_{m k}}{R_{m k}},
$$

where $J_{1}(\zeta)$ is the first-order Bessel function of the first kind, $\lambda_{a b}^{2}=\Lambda s=\lambda^{2} s / d$ is the penetration depth for the in-plane currents, and

$$
Z(q)=1+2 q \Lambda / \tanh (q s)
$$

In order to find the equilibrium form of the vortex line in a finite stack of $N$ superconducting layers under the influence of the in-plane external magnetic field $\mathbf{H}_{\|}$, we consider the relaxation of the set of the pancakes towards the equilibrium positions within the simplest version of the dynamic theory:

$$
\eta \frac{d \mathbf{r}_{n}}{d t}=\sum_{m \neq n} \mathbf{F}_{n}^{m}+\mathbf{F}_{n}^{M},
$$

where $\eta$ is the viscous drag coefficient. Considering the vortex line consisting of $N=31$ pancakes we start from the initial configuration of pancakes arranged in a straight vortex line parallel to the $z$ direction (see Fig. 2). As the system approaches its final force-balanced (equilibrium) configuration, the velocities of all pancake motions should vanish:

$$
\lim _{t \rightarrow \infty} \frac{d \mathbf{r}_{n}}{d t}=0, \quad 1 \leq n \leq N .
$$

In Fig. 2 we illustrate the evolution of the pancake configurations for two values of the applied in-plane magnetic field $\mathbf{H}_{\|}$and for two different numbers of layers: $N=31$ (Fig. 2a, 2b) and $N=11$ (Fig. 2c, 2d). The forces $\mathbf{F}_{n}^{M}$ caused by the Meissner currents rotate and 
bend the vortex line. For rather small applied field values this rotation and bending result in the formation of a certain stable configuration (see Fig. 2a, 2c). For the fields exceeding a certain critical value $H^{*}$ we do not find such stable pancake arrangement. The vortex line splits into two segments which move in opposite directions (see Fig. 2b, 2d). To define the critical value $H^{*}$ for the breakup of the vortex line we have carried out the calculations of the pancake arrangements increasing the in-plane magnetic field with the step $\delta H_{a}=0.01 H_{0}$ $\left(H_{0}=\phi_{0} / 2 \pi \lambda_{a b}^{2}\right)$. The stationary vortex arrangement disappears above a certain threshold field value which is taken as a critical field $H^{*}$. The pancake configurations for the both cases $N=31$ and $N=11$ are qualitatively similar but the values of the critical field $H^{*}$ for $N=31$ and $N=11$ are different. With a decrease in the number of layers $N$ (film thickness) the value of the critical field $H^{*}$ grows: $H^{*}=0.21 H_{0}$ for $N=31$ and $H^{*}=0.38 H_{0}$ for $N=11$. In fact in layered superconductors with very weak interlayer coupling the Josephson vortices will appear at much lower field $H_{a} \sim H_{c 1}^{(0)} \ll H^{*}$. As a result, at tilted magnetic field, crossing lattice of pancakes, forming Abrikosov vortices, and Josephson vortices exist rather than a lattice of tilted vortex $\operatorname{stacks}^{18,23,24}$. The interaction between pancakes and in-plane field in the form of Josephson vortices produces zigzag deformation of the stack of the pancakes ${ }^{25}$. This deformation is responsible for a long range attraction between such stacks $^{26}$ which is quite similar to the case of considered in the present work.

\section{B. Vortex line within anisotropic London model}

We proceed now with the consideration of the vortex line shape in an anisotropic film which is characterized by the London penetration depths $\lambda_{a b}$ and $\lambda_{c}$ for currents flowing parallel and perpendicular to the $a b$ plane, respectively. We consider the case of uniaxial anisotropy which can be described by a dimensionless anisotropic mass tensor $m_{i j}=m_{0}\left(\delta_{i j}+\right.$ $\left.\left(\Gamma^{2}-1\right) c_{i} c_{j}\right)$, where $\Gamma=\lambda_{c} / \lambda_{a b}$ is the anisotropy parameter and $\mathbf{c}$ is the anisotropy axis. We choose the $z$ axis of the coordinate system perpendicular to the film surface. In the parallel to the film plane direction we apply a certain magnetic field $\mathbf{H}_{\|}=\mathbf{y}_{0} H_{a}$ which is screened inside the superconducting film.

We consider first a typical geometry when the $\mathbf{c}$ axis is chosen along the direction normal to the film plane. In such geometry the vortex line is parallel to the plane $(y, z)$ and can be parameterized by a single valued function $y=y(z)$. An appropriate thermodynamic 
potential for determination of the energetically favorable form of the vortex line takes the form

$$
G=F_{v}-\frac{\Phi_{0}}{4 \pi} \int_{-D / 2}^{D / 2} d z\left(1-\frac{\cosh \left(z / \lambda_{a b}\right)}{\cosh \left(D / 2 \lambda_{a b}\right)}\right) y^{\prime}(z) H_{a}
$$

where $D$ is the film thickness. The first term, $F_{v}$, is the Ginzburg-Landau free energy of the curved vortex line, and the second term corresponds to the work of Lorentz force acting on the flux line and distorting this line in the presence of screening currents induced by the external magnetic-field component $H_{a}$ parallel to the film plane. To simplify the $F_{v}$ expression we consider a strong type-II superconducting material with a large ratio of the London penetration depths and coherence lengths. In this case the main contribution to the vortex line energy is determined by the energy of supercurrents $\mathbf{j}_{v}=c \operatorname{rot} \mathbf{B}_{v} / 4 \pi$ flowing around the vortex core

$$
F_{v} \simeq \frac{\lambda_{a b}^{2}}{8 \pi} \int d V \operatorname{rot} \mathbf{B}_{v}\left(\widehat{\mu} \operatorname{rot} \mathbf{B}_{v}\right) \simeq \frac{\Phi_{0}^{2}}{32 \pi^{3} \lambda_{a b}^{2}} \int d V\left(\widehat{\mu}^{-1} \nabla \theta_{v}, \nabla \theta_{v}\right)
$$

where $\widehat{\mu}=\widehat{m} / m_{0}$, and $\theta_{v}$ is the order parameter phase distribution around the vortex line. The above expression for the free energy reveals a logarithmic divergence which should be cut-off at both the small and large spatial length scales. The lower cut-off length is naturally equal to the characteristic size $r_{c}$ of the vortex core which is of the order of the coherence length. Of course, in anisotropic case one should introduce two different coherence lengths $\xi_{a b}$ and $\xi_{c}$ in the $a b$ plane and along the $c$ axis, respectively. The resulting core size and lower cut-off length $r_{c}$ for a certain element of the tilted flux line will, thus, depend on both $\xi_{a b}$ and $\xi_{c}$ lengths as well as on the local tilting angle of the vortex line. The upper cut-off length strongly depends on the ratio of the film thickness to the London penetration depth. For rather thick films $d \gg \lambda_{a b}$ this cut-off length $L_{c}$ is determined by a certain combination of the screening lengths $\lambda_{a b}$ and $\lambda_{c}$ (see, e.g., Ref. 27). For a thin film with $d \ll \lambda_{a b}$ one can separate two energy contributions: (i) the contribution coming from the region of the size $\sim d$ around the curved vortex line and providing the logarithmic term in the free energy with the upper cut-off length $L_{c} \simeq d$; (ii) the logarithmic contribution $\propto \ln \left(\lambda_{a b}^{2} / d^{2}\right)$ coming from the larger distances $\rho>d$ which weakly depends on the vortex line shape. To sum up, the part of the vortex line energy which depends on its shape can be approximately written in the form:

$$
\delta F_{v} \simeq \frac{\Phi_{0}^{2}}{16 \pi^{2} \Gamma \lambda_{a b}^{2}} \ln \left(L_{c} / r_{c}\right) \int_{-D / 2}^{D / 2} d z \sqrt{\Gamma^{2}+y^{\prime 2}(z)}
$$


Note that we neglect here the weak dependence of the logarithmic factor on the vortex line curvature and local orientation. Within such approximation we consider the vortex line as a thin elastic string which is, of course, valid provided the characteristic scale of the string bending is larger than the upper cut-off length $L_{c}$.

The condition of the zero first variation of the Gibbs functional gives us the following equation

$$
y^{\prime}(z)=\frac{\Gamma b(z)}{\sqrt{1-b^{2}(z)}}
$$

where

$$
b(z)=\frac{H_{a}}{H_{a b}} \times\left(1-\frac{\cosh \left(z / \lambda_{a b}\right)}{\cosh \left(D / 2 \lambda_{a b}\right)}\right), H_{a b}=\frac{\Phi_{0}}{4 \pi \Gamma \lambda_{a b}^{2}} \ln \left(L_{c} / r_{c}\right) .
$$

The equation (20) is valid for magnetic fields which do not exceed the critical field of the penetration of vortices parallel to the film plane

$$
\left|H_{a}\right|<H_{c 1}^{(0)}=H_{a b} \frac{\cosh \left(D / 2 \lambda_{a b}\right)}{\cosh \left(D / 2 \lambda_{a b}\right)-1} .
$$

Thus, analogously to the case of a stack of decoupled layers the stable curved vortex lines can exist only for rather small magnetic fields below the critical field $H_{c 1}^{(0)}$ which corresponds to the penetration of a vortex parallel to the film plane. Note that in the limit $H_{a} \ll H_{a b}$ one can obtain the result found previously in Ref. 14:

$$
y^{\prime}(z) \simeq \frac{\Gamma H_{a}}{H_{a b}}\left(1-\frac{\cosh \left(z / \lambda_{a b}\right)}{\cosh \left(D / 2 \lambda_{a b}\right)}\right), \quad y(z) \simeq \frac{\Gamma H_{a}}{H_{a b}}\left(z-\lambda_{a b} \frac{\sinh \left(z / \lambda_{a b}\right)}{\cosh \left(D / 2 \lambda_{a b}\right)}\right) .
$$

Typical shape of a bent vortex line calculated from Eq. (20) is shown in Fig. 3a.

The above expressions can be easily generalized for an arbitrary angle $\chi$ between the anisotropy axis $\mathbf{c}$ and the direction normal to the film plane. We restrict ourselves to the case when the axis $\mathbf{c}$ is parallel to the plane $(y, z)$ and vortex line can be parameterized by a function $y=y(z)$ as before. In this case the part of the free energy (18) depending on the vortex line shape takes the form:

$$
\delta F_{v} \simeq \frac{\Phi_{0}^{2}}{16 \pi^{2} \Gamma \lambda_{a b}^{2}} \ln \left(L_{c} / r_{c}\right) \int_{-D / 2}^{D / 2} d z \sqrt{1+y^{\prime 2}(z)} \sqrt{\sin ^{2} \theta(\mathrm{z})+\Gamma^{2} \cos ^{2} \theta(\mathrm{z})}
$$

where $\tan [\theta(z)+\chi]=y^{\prime}(z)$. Thus we find the following equation describing the vortex line shape:

$$
y^{\prime}(z)=\frac{t\left(1-\Gamma^{2}\right)}{1+t^{2} \Gamma^{2}}+\frac{\Gamma N(z)\left(1+t^{2}\right)}{\left(1+t^{2} \Gamma^{2}\right) \sqrt{1+t^{2} \Gamma^{2}-N^{2}(z)}},
$$


where

$$
N(z)=\frac{H_{a}}{H_{a b}} \sqrt{1+t^{2}} \times\left(1-\frac{\cosh \left(\mathrm{z} / \lambda_{\mathrm{ab}}\right)}{\cosh \left(\mathrm{D} / 2 \lambda_{\mathrm{ab}}\right)}\right), t=\tan \chi .
$$

Note that the equation (22) is valid in the field range

$$
\left|H_{a}\right|<H_{c 1}^{(\chi)}=\frac{H_{a b} \cosh \left(D / 2 \lambda_{a b}\right)}{\cosh \left(D / 2 \lambda_{a b}\right)-1} \sqrt{\frac{1+t^{2} \Gamma^{2}}{1+t^{2}}} .
$$

The critical field $H_{c 1}^{(\chi)}$ corresponds to the penetration of a vortex parallel to the film plane. Typical plots illustrating the numerical solution of the equation (22) are shown in the Fig. 3b for different orientations of the applied magnetic field. Note an important difference between the opposite directions of the magnetic field $H_{a}$ : for $H_{a}>0$ the vortex line consists of segments tilted in opposite directions with respect to the $z$ axis.

\section{VORTEX-VORTEX INTERACTION POTENTIAL}

In this section we derive general expressions for the interaction energy between two vortices in a thin film of an anisotropic superconductor taking into account both long range attraction and repulsion phenomena. We study both the limits of strong and moderate anisotropy for a wide range of vortex tilting angles. The shape of the interacting vortex lines is assumed to be fixed and not affected by the vortex-vortex interaction potential. Certainly, such assumption is valid only in the limit of rather larger distances between the vortex lines when the effect of interaction on the vortex shape can be viewed as a small perturbation.

\section{A. Interaction potential of two tilted stacks of pancakes}

In this section we consider the interaction between two vortex lines consisting of pancake vortices. For each vortex the pancake centers are assumed to be positioned along the straight line tilted at the angle $\gamma$ with respect to the anisotropy axis $\mathbf{c}(z$ axis). We restrict ourselves to the case of parallel vortex lines shifted by a certain vector $\mathbf{R}$ in the plane of the layers. Using the gauge $\operatorname{div} \mathbf{A}=0$ and the Fourier transform

$$
\begin{gathered}
\mathbf{A}(\mathbf{q}, k)=\int d^{2} \mathbf{r} d z \mathrm{e}^{i \mathbf{q} \mathbf{r}+i k z} \mathbf{A}(\mathbf{r}, z) \\
\mathbf{A}_{n}(\mathbf{q})=\int d^{2} \mathbf{r} \mathrm{e}^{i \mathbf{q} \mathbf{r}} \mathbf{A}\left(\mathbf{r}, z_{n}\right), \quad \mathbf{J}_{n}(\mathbf{q})=\int d^{2} \mathbf{r} \mathrm{e}^{i \mathbf{q r}} \mathbf{J}_{n}(\mathbf{r}),
\end{gathered}
$$


one can rewrite the basic equation (1) in the momentum representation as follows:

$$
\left(q^{2}+k^{2}\right) \mathbf{A}(\mathbf{q}, k)=\frac{1}{\Lambda} \sum_{n}\left(\mathbf{\Phi}_{n}(\mathbf{q})-\mathbf{A}_{n}(\mathbf{q})\right) \mathrm{e}^{i k n s}
$$

where $\boldsymbol{\Phi}_{n}(\mathbf{q})=\boldsymbol{\Phi}(\mathbf{q}) \mathrm{e}^{i \mathbf{q} \mathbf{r}_{n}}$. Taking account of the relation

$$
2 \pi \mathbf{A}_{n}(\mathbf{q})=\int d k \mathrm{e}^{i k z_{n}} \mathbf{A}(\mathbf{q}, k)
$$

we obtain from (26) the following equations for the Fourier components of the vector potential $\mathbf{A}_{n}(\mathbf{q})$ :

$$
2 q \Lambda \mathbf{A}_{n}=\sum_{m}\left(\mathrm{e}^{i \mathbf{q} \mathbf{r}_{m}} \boldsymbol{\Phi}(\mathbf{q})-\mathbf{A}_{m}\right) \mathrm{e}^{-|n-m| q s} .
$$

These equations can be reduced to the scalar form

$$
f_{n}+\frac{1}{2 q \Lambda} \sum_{m} \mathbf{e}^{-|n-m| q s} f_{m}=\mathbf{e}^{i \mathbf{q r} \mathbf{r}_{n}}
$$

where we introduce the new functions $f_{n}(\mathbf{q})$ :

$$
\mathbf{J}_{n}(\mathbf{q})=\frac{c}{4 \pi \Lambda}\left(\mathbf{\Phi}_{n}(\mathbf{q})-\mathbf{A}_{n}(\mathbf{q})\right)=\frac{c}{4 \pi \Lambda} \mathbf{\Phi}(\mathbf{q}) f_{n}(\mathbf{q})
$$

The solution of the linear system (28) for a fixed distribution of pancakes $\mathbf{r}_{n}$ determines the distribution of the vector potential $\mathbf{A}(\mathbf{r}, z)$ which is created by an arbitrary vortex line in a finite stack of superconducting layers. In the momentum representation the general expression (44) for the free energy of the layered system without Josephson coupling reads

$$
F=\frac{1}{32 \pi^{3} \Lambda} \sum_{n} \int d^{2} \mathbf{q}\left(\boldsymbol{\Phi}_{n}(\mathbf{q})-\mathbf{A}_{n}(\mathbf{q})\right) \mathbf{\Phi}_{n}(-\mathbf{q})
$$

For two vortex lines we can write the total vector potential and the total sheet current as superpositions of contributions coming from the first $\left(\mathbf{A}_{n}^{(1)}, \mathbf{J}_{n}^{(1)}\right)$ and second $\left(\mathbf{A}_{n}^{(2)}, \mathbf{J}_{n}^{(2)}\right)$ vortices:

$$
\mathbf{A}_{n}(\mathbf{q})=\mathbf{A}_{n}^{(1)}(\mathbf{q})+\mathbf{A}_{n}^{(2)}(\mathbf{q}), \quad \mathbf{J}_{n}(\mathbf{q})=\mathbf{J}_{n}^{(1)}(\mathbf{q})+\mathbf{J}_{n}^{(2)}(\mathbf{q})
$$

Calculating the interaction energy $\varepsilon_{\text {int }}$ of vortex lines we should keep in (30) only the terms which contain the products of fields corresponding to different vortex lines:

$\varepsilon_{i n t}=\frac{1}{32 \pi^{3} \Lambda} \sum_{n} \int d^{2} \mathbf{q}\left[\left(\boldsymbol{\Phi}_{n}^{(1)}(\mathbf{q})-\mathbf{A}_{n}^{(1)}(\mathbf{q})\right) \boldsymbol{\Phi}_{n}^{(2)}(-\mathbf{q})+\left(\boldsymbol{\Phi}_{n}^{(2)}(\mathbf{q})-\mathbf{A}_{n}^{(2)}(\mathbf{q})\right) \boldsymbol{\Phi}_{n}^{(1)}(-\mathbf{q})\right]$. 
Finally, for the particular case of two parallel vortex lines which are shifted at the vector $\mathbf{R}=\mathbf{r}_{n}^{(2)}-\mathbf{r}_{n}^{(1)}(n=1, N)$ in the $(x y)$ plane we get following expression for the interaction energy via the scalar functions $f_{n}(\mathbf{q})$ :

$$
\varepsilon_{i n t}(\mathbf{R})=\frac{\phi_{0}^{2}}{16 \pi^{3} \Lambda} \int \frac{d^{2} \mathbf{q}}{q^{2}} \cos (\mathbf{q} \mathbf{R}) \sum_{n} f_{n}(\mathbf{q}) \mathrm{e}^{-i \mathbf{q r}_{n}} .
$$

The expression (32) and equations (28) determine the interaction energy of two identically bent vortex lines.

Our further consideration in this subsection is based on two assumptions: (i) for each vortex we choose the centers of pancakes to be positioned along the straight line tilted at a certain angle $\gamma$ relative to $z$ axis, and put $\mathbf{r}_{n}=n s \tan \gamma \mathbf{y}_{0}$; (ii) we restrict ourselves by the continuous limit assuming $q s \ll 1$ and $q_{y} s \tan \gamma \ll 1$. In this case the Eqs. (28), (32) can be simplified (see Appendix @ for details):

$$
\begin{aligned}
& \varepsilon_{\text {int }}(\mathbf{R})=\frac{\phi_{0}^{2}}{16 \pi^{3} \lambda_{a b}} \int d^{2} \mathbf{q} \cos (\mathbf{q} \mathbf{R}) S(\mathbf{q}), \\
& S(\mathbf{q})=\frac{1}{\lambda_{a b} q^{2}}\left\{D \frac{p^{2}+k^{2}}{1+p^{2}}\right. \\
& \left.\quad+\frac{2\left(1-k^{2}\right)\left[k\left(1-p^{2}\right) \sinh L+\left(k^{2}-p^{2}\right)(\cosh L-\cos (p L))+2 k p \sin (p L)\right]}{\left.\sqrt{q^{2}+\lambda_{a b}^{-2}}\left(1+p^{2}\right)^{2}\left[2 k \cosh L+\left(1+k^{2}\right) \sinh L\right)\right]}\right\},
\end{aligned}
$$

where

$$
L=D \sqrt{q^{2}+\lambda_{a b}^{-2}}, \quad k=q / \sqrt{q^{2}+\lambda_{a b}^{-2}}, \quad p=q_{y} \tan \gamma / \sqrt{q^{2}+\lambda_{a b}^{-2}},
$$

and $D=(N-1) s$ is the thickness of the superconducting film. The first term in (34) describes the interaction in the bulk system, while the second term is responsible for the effect of film boundaries.

The minimum energy configuration corresponds to the case $R_{x}=0$. In Fig. 4 we present some typical plots of the interaction energy $\varepsilon_{i n t}\left(R_{x}=0, R_{y}\right)$ vs the distance $R_{y}=R$ for $d=3 \lambda_{a b}$ which corresponds to the Lorentz microscopy experiments in $\mathrm{YBCO}^{\underline{\underline{5}}}$ and $\mathrm{Bi}-2212^{20}$ samples. Analyzing the dependence $\varepsilon_{\text {int }}(R)$, one can separate three contributions to the energy of vortex-vortex interaction: (i) a short-range repulsion which decays exponentially with increasing intervortex distance $R$ (for $R>\lambda_{a b}$ ); (ii) an intervortex attraction which is known to be specific for tilted vortices in anisotropic systems; this attraction energy term decays as $R^{-2}$ and strongly depends on the angle $\gamma$ between the vortex axis and the c direction; (iii) long-range (Pearl) repulsion which decays as $R^{-1}$ and results from the 
surface contribution to the energy. Note that the third term does exist even for a large sample thickness $D$ (see Ref. ${ }^{28}$ ) although in the limit $D \gg \lambda_{a b}$ it is certainly masked by the dominant bulk contribution. At $R \gg \lambda_{a b}$ the short-range interaction term vanishes and the interaction energy vs $R$ takes the form

$$
\varepsilon_{\text {int }} \simeq \frac{\phi_{0}^{2}}{8 \pi^{2}}\left(-\frac{D_{e f f} \tan ^{2} \gamma}{R^{2}}+\frac{2}{R}\right)
$$

where $D_{\text {eff }}=D-2 \lambda_{a b} \tanh \left(D / 2 \lambda_{a b}\right)$ is the effective film thickness. One can observe here an interplay between the long-range attractive (first term in Eq. (36) ) and the repulsive (second term in Eq.(36) ) forces. Note that the $\lambda_{a b}$ value increases with an increase in temperature, thus, the effective thickness decreases and the long range attraction force appears to be suppressed with increasing temperature. For large $R$ the energy is always positive and corresponds to the vortex repulsion similar to the one between the pancakes in a single layer system. With a decrease in the distance $R$ the attraction force comes into play resulting in the change of the sign of the energy. Such behavior points to the appearance of a minimum in the interaction potential.

\section{B. Vortex-vortex interaction within anisotropic London model}

We now proceed with the derivation of the expression for the intervortex interaction energy in an anisotropic superconducting film. We choose the anisotropy axis $\mathbf{c}(z-$ axis) to be oriented perpendicular to the film plane and consider two curved vortex lines with identical shapes found in Sec. II B. Our further calculations are based on general expressions derived in Ref. 29 for the energy of an arbitrary vorticity distribution in an anisotropic superconducting film of finite thickness (see Appendix B for details). The resulting interaction energy of two curved vortices shifted from each other in the $y$ direction at a certain distance $R$ can be presented in the form:

$$
\varepsilon_{i n t}=\varepsilon_{0}\left(\epsilon_{i n t}^{v i}+\epsilon_{i n t}^{s t r a y}+\epsilon_{i n t}^{v a c}\right)
$$

where $\varepsilon_{0}=\phi_{0}^{2} / 16 \pi^{3} \lambda_{a b}$, while $\epsilon_{i n t}^{v i}, \epsilon_{i n t}^{\text {stray }}$, and $\epsilon_{\text {int }}^{v a c}$ are given by the expressions (B5), (B6), (B7).

Considering the particular case of straight vortex lines parallel to the plane $(y z)$ and tilted at a certain angle $\gamma$ with respect to the $\mathbf{c}$ direction we obtain the following expression 
for the interaction energy of two vortices:

$$
\begin{aligned}
\varepsilon_{i n t}(\mathbf{R}) & =\frac{\phi_{0}^{2}}{16 \pi^{3} \lambda_{a b}} \int d^{2} \mathbf{q} \cos (\mathbf{q} \mathbf{R}) S_{\Gamma}(\mathbf{q}) \\
S_{\Gamma}(\mathbf{q}) & =\frac{1}{\lambda_{a b} q^{2}}\left\{D\left(\frac{1+k_{\Gamma}^{2}}{1+p_{\Gamma}^{2}}-\frac{1}{\left(1+q^{2}\right)\left(1+p^{2}\right)}\right)\right. \\
+ & \left.\frac{2\left(1-k^{2}\right)\left[k\left(1-p^{2}\right) \sinh L+\left(k^{2}-p^{2}\right)(\cosh L-\cos (p L))+2 k p \sin (p L)\right]}{\left.\sqrt{q^{2}+\lambda_{a b}^{-2}}\left(1+p^{2}\right)^{2}\left[2 k \cosh L+\left(1+k^{2}\right) \sinh L\right)\right]}\right\}
\end{aligned}
$$

where

$$
k_{\Gamma}=q \tan \gamma / \sqrt{\Gamma^{2} q^{2}+\lambda_{a b}^{-2}}, \quad p_{\Gamma}=q_{y} \tan \gamma / \sqrt{\Gamma^{2} q^{2}+\lambda_{a b}^{-2}},
$$

and the parameters $L, k$ and $p$ are described by the expressions (35). In the limit of strong anisotropy $(\Gamma>>1)$ the spectral function $S_{\Gamma}(\mathbf{q})$ (39) naturally coincides with the corresponding expressions (34) obtained for the layered system without Josephson coupling.

Some typical plots of the interaction energy vs the intervortex distance for tilted vortex lines calculated using the Eqs. (38), (39) are shown in Figs. 5, 6, Analyzing the dependence $\varepsilon_{\text {int }}(R)$ one can separate three contributions to the energy of intervortex interaction: (i) a short-range repulsion (for $R \ll \lambda_{a b} \sqrt{1+\tan ^{2} \gamma}$ ) which decays exponentially with increasing intervortex distance $R$; (ii) an intervortex attraction which comes into play for the region $\lambda_{a b} \sqrt{1+\tan ^{2} \gamma}<R<\Gamma \lambda_{a b}$ and decays exponentially with the vortex-vortex distance $R$ for $R>\Gamma \lambda_{a b}$; (iii) long-range (Pearl) repulsion which decays as $R^{-1}$ at large distances and results from the surface contribution to the energy. Taking the limit $R \ll \lambda_{a b} \sqrt{1+\tan ^{2} \gamma}$ we get

$$
\varepsilon_{i n t} / \varepsilon_{0} \simeq \frac{D \pi \sqrt{\Gamma^{2}+\tan ^{2} \gamma}}{\Gamma} \ln \left(\frac{L_{c}}{R}\right) .
$$

In the region $\lambda_{a b} \sqrt{1+\tan ^{2} \gamma}<R<\Gamma \lambda_{a b}$ the short-range interaction term vanishes and the interaction energy vs $R$ is given by the sum (36) of attractive and Pearl's contributions. Similarly to the case of decoupled layers discussed above the attractive term can result in the appearance of a minimum in the dependence of the vortex-vortex interaction potential vs $R$. The position of this minimum can be roughly estimated as the boundary of the region of the short-range repulsion: $R_{\text {min }} \simeq \lambda_{a b} \sqrt{1+\tan ^{2} \gamma}$. Obviously, the minimum should disappear provided $R_{\min }>\Gamma \lambda_{a b}$, i.e., when the region of the attraction between vortices vanishes. This condition gives us the the upper boundary on the tilting angle $\gamma$ restricting the interval 
of the energy minimum existence:

$$
\tan ^{2} \gamma<\Gamma^{2}-1 .
$$

The lower boundary of this angular interval can be found comparing the attractive and repulsive terms in the expression (36) at the distance $R_{\text {min }}$ :

$$
\tan ^{2} \gamma>\frac{2 \lambda_{a b}^{2}}{D_{e f f}^{2}}\left(1+\sqrt{1+\frac{D_{e f f}^{2}}{\lambda_{a b}^{2}}}\right) .
$$

These analytical estimates of the angular interval are in a rough qualitative agreement with the numerical calculations (see Figs. [5],6) for two values of the film thickness $D=3 \lambda_{a b}, 10 \lambda_{a b}$. Indeed, one can see that increasing the tilting angle we first deepen the minimum in the interaction potential and then make it more shallow. The figures confirms the deepening of the minimum with the increase in the anisotropy parameter $\Gamma$. Our numerical calculations demonstrate that for the film thickness $D=3 \lambda_{a b}$ (Fig. (5) the minimum of the interaction energy of two straight tilted vortices can appear only for $\Gamma \gtrsim 14$. Starting from $\Gamma \approx 27$ the bound vortex pair becomes energetically favorable. An increase in the film thickness reduces the relative contribution of Pearl repulsion to the energy of intervortex interaction $\varepsilon_{\text {int }}$. As a result attraction of vortices takes place for smaller values of the tilting angle $\gamma$ and anisotropy parameter $\Gamma$. Thus, in a film with the thickness $D=10 \lambda_{a b}$ (Fig. 66) the minimum in the $\varepsilon_{\text {int }}(R)$ dependence appears for $\Gamma \gtrsim 7$, whereas creation of the bound vortex pair becomes energetically favorable for $\Gamma \gtrsim 9$.

As a next step, we check if the above results obtained for straight tilted vortices remain valid for the curved vortex lines. Our analysis of the effect of the vortex line curvature is carried out for model vortex profiles found in Sec. II B. The resulting typical dependencies of the intervortex interaction potential vs $R$ for different magnetic field values and anisotropy parameters are shown in Figs. 778, One can clearly see that the minimum in the interaction potential vs $R$ survives when we take account of the vortex line curvature. Moreover the curving of the vortex line even deepens this minimum as it is confirmed by the comparison of energies of straight tilted and curved vortices presented in Fig. 8, For such comparison we choose the straight vortex lines connecting the ends of curved vortices. We find that for curved vortices the energy minimum exists even for smaller anisotropy parameters than for straight vortices (i.e., the threshold anisotropy value for $D=3 \lambda_{a b}$ becomes less than 
$\Gamma \approx 14$ ). Of course, increasing the film thickness one can weaken the restrictions on the existence of the minimum in the interaction potential: e.g., for $D=10 \lambda_{a b}$ the minimum appears at $\Gamma \gtrsim 9$.

The above theoretical analysis demonstrates that vortex-vortex attraction and the formation of chains are possible only for the rather large tilting angles and at low vortex concentrations, i.e., when the magnetic-field component $H_{z}$ perpendicular to the film plane is very weak. In fields $H_{z}$ slightly above $H_{c 1}$ Abrikosov vortices will form chains due to the long range attractive interaction. Peculiarities of penetration of such chains of tilted Abrikosov vortices into bulk layered (anisotropic) superconductor are well known: in the

first approximation, the vortex period in chains does not depend on applied magnetic field, while the distance between chains changes as $1 / H_{z}$. The presence of vortex chains significantly modifies the magnetization curves with respect to analogous curves for isotropic superconductors. ${ }^{30}$ In the next sections we discuss additional peculiarities of intervortex interaction specific for thin-film samples of layered (anisotropic) superconductors.

\section{VORTEX CLUSTERS}

The unusual vortex-vortex interaction potential behavior discussed in the previous section can result in unconventional vortex structures. We start our analysis of energetically favorable vortex structures from the problem of stability of a vortex chain. The formation of infinite vortex chains is known to be a signature of the intervortex attraction in bulk anisotropic superconductors. The long range repulsion of vortices in thin films can destroy the infinite vortex chains. Indeed, despite of the fact that two vortices attract each other at a certain distance, further increase in the number of vortices arranged in a chain can be energetically unfavorable because of the slower decay of the repulsive force compared to the attractive one. In this case, for rather thin samples, there appears an intriguing possibility to observe vortex chains of finite length, i.e., vortex molecules or clusters. In this section we present the calculations of energies of such vortex clusters.

As we have demonstrated above, the minimum in the interaction potential exists for both the limits of strong and moderate anisotropy. The vortex molecule cohesion energy is given 
by the expression:

$$
\varepsilon_{i n t}^{(N)}=\sum_{i>j} \varepsilon_{i n t}\left(R_{i j}\right)
$$

where $N$ is the number of vortices in the molecule, and $R_{i j}$ are the distances between $i$-th and $j$-th vortices in the chain molecule. Shown in Figs. 9,10 are typical plots of the interaction energy per vortex vs the intervortex distance $R$ for equidistant vortex chains with different $N$ numbers calculated within the model of decoupled superconducting layers and anisotropic London theory. The energetically favorable number of vortices in a molecule grows as we increase the film thickness and/or the tilting angle because of the increasing attraction term in the pair potential $\varepsilon_{i n t}$. Shown in the insets of Figs. 9 are schematic pictures of vortex matter consisting of dimeric and trimeric molecules. Finally, for rather thick samples with $D \gg \lambda_{a b}$ we get a standard infinite chain structure typical for bulk systems. Note that the formation of an infinite vortex chain may be considered in some sense as a polymerization of the vortex molecules. Certainly, the crossover from the vortex molecule state to the infinite chain structure is strongly influenced by the increase in the vortex concentration governed by the component $B_{z}$ of the external magnetic field perpendicular to the film. Indeed, one can expect such a cross-over to occur when the mean intervortex spacing approaches the molecule size. Thus, the vortex molecule state can appear only in a rather weak perpendicular field when its observation can be complicated, of course, by the pinning effects.

\section{PHASE TRANSITIONS IN VORTEX LATTICES}

Considering the effect of a finite magnetic field (i.e., a finite concentration of vortex clusters) we restrict ourselves by the simplest case of regular vortex arrays. For a regular vortex array the formation of clusters corresponds to the transition with a change in the number of vortices in the elementary lattice cell. The mechanism underlying such transition is naturally connected with the appearance of the minimum in the interaction potential for a vortex pair. In this section we present our calculations of energy of vortex lattices with different number of flux quanta per unit cell. The possibility to get the energetically favorable states with a few vortices per unit cell will be illustrated for a particular intervortex

interaction potential derived above for a model of decoupled superconducting layers. The generalization of such consideration for anisotropic London theory is straightforward. Note 
that the vortex lattice structure for bulk anisotropic superconductors in tilted field in the framework of London approach has been calculated in Ref. 31 .

Let's consider a vortex lattice characterized by the translation vectors $\mathbf{T}=i \mathbf{a}_{1}+j \mathbf{a}_{2}$, where $\mathbf{a}_{1,2}$ are primitive vectors of the lattice. The primitive cell occupies the area $A_{0}=$ $\left[\mathbf{a}_{1} \times \mathbf{a}_{2}\right] \cdot \mathbf{z}_{0}$ and is assumed to contain $M$ vortices: $B_{z} A_{0}=M \Phi_{0}$. Positions of vortices in a cell are determined by the vectors $\mathbf{r}_{m}(m=1, M)$ (see Fig. 111). The interaction energy per unit lattice cell can be expressed via the vortex-vortex interaction potentials (33), (34):

$$
\varepsilon_{c}\left(\mathbf{r}_{m k}, \mathbf{T}\right)=\sum_{m, k \neq m}^{M} \varepsilon_{i n t}\left(\mathbf{r}_{m k}\right)+\sum_{\mathbf{T} \neq 0} \sum_{m, k}^{M} \varepsilon_{i n t}\left(\mathbf{T}+\mathbf{r}_{m k}\right) .
$$

The interaction energy (41) depends on both the relative positions $\mathbf{r}_{m k}=\mathbf{r}_{m}-\mathbf{r}_{k}$ of vortices in the primitive cell and the structure of the vortex lattice defined by the translation vectors $\mathbf{T}$. The first term in (41) describes the interaction energy between vortices in the primitive cell (without the lattice contribution), whereas the second sum takes account of the lattice effects. With the help of the Poisson formula, one can rewrite the intervortex interaction energy (41) in terms of the Fourier components

$$
\varepsilon_{c}=\frac{\phi_{0}^{2}}{16 \pi^{3} \lambda_{a b}}\left[\frac{4 \pi^{2}}{A_{0}} \sum_{\mathbf{Q}} \sum_{m, k}^{M} S(\mathbf{Q}) \cos \left(\mathbf{Q} \mathbf{r}_{m k}\right)-M \int d^{2} \mathbf{q} S(\mathbf{q})\right],
$$

where the function $S(\mathbf{q})$ is determined by the Eq. (34), and $\mathbf{Q}$ are the reciprocal-lattice vectors. The sum and the integral in Eq. (42) diverge both at $\mathbf{Q}=0$ and at large $\mathbf{Q}$ values. The small $\mathbf{Q}$ divergence corresponds to the linear (in the system size) increase in the vortex energy because of the slow $1 / R$ decay of the vortex-vortex interaction potential. The large Q divergence is logarithmic and is associated with the vortex self energy.

For simplicity, we restrict ourselves to the case of an instability with respect to the unit cell doubling and tripling, i.e. formation of the vortex lattices with two and three flux quanta per unit cell $(M=2$ and $M=3)$. Hereafter we consider only the shifts of vortex sublattices along the $y$ direction and choose the appropriate reciprocal-lattice vectors

$$
\begin{aligned}
& \mathbf{Q}_{i j}=\frac{2 \pi}{b}(i-j / 4) \mathbf{x}_{0}+\frac{\pi}{a} j \mathbf{y}_{0}, \quad i, j=0, \pm 1, \pm 2, \ldots, \\
& \mathbf{Q}_{i j}=\frac{2 \pi}{b}(i-j / 6) \mathbf{x}_{0}+\frac{2 \pi}{3 a} j \mathbf{y}_{0}, \quad i, j=0, \pm 1, \pm 2, \ldots
\end{aligned}
$$

for $M=2$ and $M=3$, respectively. Here we consider only equidistant vortex chains within the primitive cells. Fixing the value of the field $B_{z}$ we fix the unit cell area area $A_{0}=2 a b$ 
for $M=2$ and $A_{0}=3 a b$ for $M=3$. Thus, the interaction energy (42) depends only on two parameters: (i) $\sigma=b / a$ ratio characterizing the lattice deformation; (ii) relative displacement $\Delta a$ of vortex sublattices along the $y$-axis (see Fig. 11). To exclude the divergence at $Q=0$ it is convenient to deal with the energy difference:

$$
\Delta \varepsilon_{c}=\min _{\sigma}\left\{\varepsilon_{c}(\sigma, \Delta a)\right\}-\min _{\sigma}\left\{\varepsilon_{c}(\sigma, 0)\right\} .
$$

The results of our numerical calculations of this energy difference are shown in Fig. 12a. One can clearly observe that changing the vortex tilting angle we obtain the minimum in the function $\Delta \varepsilon_{c}(\Delta a)$ which gives us the evidence for the phase transition in the lattice structure with the unit cell doubling or tripling depending on the vortex tilting angle. The multiplication of the unit cell is accompanied by the strong change in the lattice deformation ratio $\sigma$ (see Fig. 12b).

\section{CONCLUSIONS}

To sum up, we suggest a scenario of the phase transitions between the flux structures with different number of vortices per unit cell which can be realized in thin films of anisotropic superconductors placed in tilted magnetic fields. We demonstrate that the vortex interaction in the films of anisotropic superconductors placed in tilted magnetic fields is very special. The underlying physics arises from the interplay between the long range attraction and repulsion between tilted vortex lines. In consequence, new and very reach types of vortex structures may appear. They are formed from the vortex dimers, trimers, etc., and the transition between different types of vortex structures may be controlled by tilting of external magnetic field and/or by varying of the temperature. Our theoretical findings are based on two theoretical approaches: anisotropic London model and the London-type model of decoupled superconducting layers. Taking account of the vortex tilt and bending we analyzed the distinctive features of the vortex-vortex interaction potential in a wide range of parameters and fields and demonstrated the possibility to obtain a minimum in the vortex interaction potential vs the intervortex distance. Further analysis in the paper included the calculations of energy of finite size vortex clusters as well as the energy of regular vortex arrays with different number of vortices per unit cell. The phase transitions accompanied by the multiplication of the primitive lattice cell appear to be possible for 
dilute vortex arrays, i.e. for rather small magnetic field component $B_{z}$. We believe that our theoretical predictions concerning the unusual vortex configurations are experimentally observable using the modern vortex imaging methods such as Lorentz microscopy, scanning tunneling microscopy, scanning Hall-probe or decoration technique.

\section{Acknowledgments}

We are grateful to Professor A. Tonomura for stimulating discussions. This work was supported, in part, by the Russian Foundation for Basic Research, Russian Academy of Sciences under the Program "Quantum physics of condensed matter", Russian Agency of Education under the Federal Program "Scientific and educational personnel of innovative Russia in 2009-2013", and "Dynasty" foundation.

\section{Appendix A: Interaction energy of tilted vortices: continuous limit}

Let us evaluate the interaction energy (32) of two tilted parallel vortex lines taking

$$
\mathbf{r}_{n}^{(1)}=n s \tan \gamma \mathbf{x}_{0}, \quad \mathbf{r}_{n}^{(2)}=\mathbf{r}_{n}^{(1)}+\mathbf{R}
$$

and assuming $q s \ll 1$ and $q_{x} s \tan \gamma \ll 1$. We introduce a continuous coordinate $t=n s$ and

continuous function $f_{\mathbf{q}}(t)$. Thus, the linear system of equations (28) reduces to the following integral equation

$$
f_{\mathbf{q}}(t)+\frac{1}{2 q \lambda_{a b}^{2}} \int_{-D / 2}^{D / 2} d t^{\prime} \mathrm{e}^{-q\left|t-t^{\prime}\right|} f_{\mathbf{q}}\left(t^{\prime}\right)=\mathrm{e}^{i q_{x} t \tan \gamma} .
$$

The equation (A1) can be rewritten as a differential one

$$
\frac{d^{2} f_{\mathbf{q}}}{d t^{2}}-\left(\lambda_{a b}^{-2}+q^{2}\right) f_{\mathbf{q}}(t)=-\left(q_{x}^{2} \tan ^{2} \gamma+q^{2}\right) \mathrm{e}^{i q_{x} t \tan \gamma}
$$

at the interval $-D / 2<t<D / 2$ with the boundary conditions

$$
\left.\left(\frac{d f_{\mathbf{q}}}{d t} \pm q f_{\mathbf{q}}\right)\right|_{ \pm D / 2}=\left(i q_{x} \tan \gamma \pm q\right) e^{ \pm i q_{x} D \tan \gamma / 2}
$$

Introducing the notations

$$
\tau=t \sqrt{q^{2}+\lambda_{\|}^{-2}}, \quad L=D \sqrt{q^{2}+\lambda_{\|}^{-2}}, \quad k=q / \sqrt{q^{2}+\lambda_{\|}^{-2}}, \quad p=q_{x} \tan \gamma / \sqrt{q^{2}+\lambda_{\|}^{-2}},
$$


one can rewrite the equation (A2) and boundary conditions (A3) in dimensionless form

$$
\begin{aligned}
& \frac{d^{2} f_{\mathbf{q}}}{d \tau^{2}}-f_{\mathbf{q}}=-\left(p^{2}+k^{2}\right) \mathrm{e}^{i p \tau} \\
& \left.\left(\frac{d f_{\mathbf{q}}}{d \tau} \pm k f_{\mathbf{q}}\right)\right|_{ \pm L / 2}=(i p \pm k) \mathrm{e}^{i p L / 2}
\end{aligned}
$$

The solution of the Eq. (A4) has the form

$$
f_{\mathbf{q}}(\tau)=\frac{p^{2}+k^{2}}{1+p^{2}} \mathrm{e}^{i p \tau}+\left(1-\frac{p^{2}+k^{2}}{1+p^{2}}\right)\left(a \mathrm{e}^{\tau}+b \mathrm{e}^{-\tau}\right)
$$

where the constants $a$ and $b$ are defined by the boundary conditions (A5):

$$
\begin{aligned}
& a=\frac{\mathrm{e}^{(i p+1) L / 2}(k+i p)(1+k)+\mathrm{e}^{-(i p+1) L / 2}(k-i p)(1-k)}{2\left(2 k \cosh L+\left(1+k^{2}\right) \sinh L\right)} \\
& b=\frac{\mathrm{e}^{(i p-1) L / 2}(k+i p)(1-k)+\mathrm{e}^{(-i p+1) L / 2}(k-i p)(1+k)}{2\left(2 k \cosh L+\left(1+k^{2}\right) \sinh L\right)} .
\end{aligned}
$$

In the continuous limit the expression for the interaction energy (32) takes the form:

$$
\varepsilon_{i n t}=\frac{\phi_{0}^{2}}{16 \pi^{3} \lambda_{a b}^{2}} \int \frac{d^{2} \mathbf{q}}{q^{2}} \cos (\mathbf{q} \mathbf{R}) S(\mathbf{q})
$$

Here the function

$$
S(\mathbf{q})=\int_{-D / 2}^{D / 2} d t f_{\mathbf{q}}(t) \mathrm{e}^{-i q_{x} t \tan \gamma}
$$

can be calculated analytically:

$$
S(\mathbf{q})=D \frac{p^{2}+k^{2}}{1+p^{2}}+\frac{2\left(1-k^{2}\right)\left[k\left(1-p^{2}\right) \sinh L+\left(k^{2}-p^{2}\right)(\cosh L-\cos (p L))+2 k p \sin (p L)\right]}{\sqrt{q^{2}+\lambda_{a b}^{-2}}\left(1+p^{2}\right)^{2}\left[2 k \cosh L+\left(1+k^{2}\right) \sinh L\right]} .
$$

\section{Appendix B: Interaction energy of curved vortices: anisotropic London model}

To calculate the vortex-vortex interaction within anisotropic London model we use general expressions derived in Ref. 29 for the total energy $E$ of an arbitrary arrangement of curved vortices in a superconducting film of thickness $D$ with the $c$-axis perpendicular to the film plane:

$$
E=E_{v i}+E_{\text {stray }}+E_{v a c}
$$

where 


$$
\begin{gathered}
E_{v i}=\frac{\Phi_{0}^{2}}{16 \pi} \int \frac{d^{2} k_{\perp}}{4 \pi^{2}} \frac{1}{2 D} \sum_{m} \sum_{\alpha} G_{\alpha}\left(\mathbf{k}_{\perp}, k_{m}\right)\left|\nu_{\alpha}^{v i}\left(\mathbf{k}_{\perp}, k_{m}\right)\right|^{2}, \\
\left.E_{\text {stray }}=\frac{1}{8 \pi} \int \frac{d^{2} k_{\perp}}{4 \pi^{2}} \frac{k_{\perp}^{2}}{v}\left[\left(1-e^{-2 v D}\right)\left|\gamma^{+}\right|^{2}+\left(e^{2 v D}-1\right)\left|\gamma^{-}\right|^{2}\right)\right], \\
E_{v a c}=\frac{1}{8 \pi} \int \frac{d^{2} k_{\perp}}{4 \pi^{2}} k_{\perp}\left(e^{-2 k_{\perp} D}\left|\phi^{-}\right|^{2}+\left|\phi^{+}\right|^{2}\right) .
\end{gathered}
$$

Here $\alpha=x, y, z$ and

$$
\begin{aligned}
& G_{x}(\mathbf{k})=G_{y}(\mathbf{k})=\frac{1}{1+k_{\perp}^{2} \lambda_{c}^{2}+k_{z}^{2} \lambda_{a b}^{2}}, G_{z}(\mathbf{k})=\frac{1+k^{2} \lambda_{c}^{2}}{\left(1+k^{2} \lambda_{a b}^{2}\right)\left(1+k_{\perp}^{2} \lambda_{c}^{2}+k_{z}^{2} \lambda_{a b}^{2}\right)}, \\
& \gamma^{-}\left(\mathbf{k}_{\perp}\right)=\frac{v\left[A\left(k_{\perp}-v\right) e^{-v D}-B\left(k_{\perp}+v\right)\right]}{k_{\perp} C}, \gamma^{+}\left(\mathbf{k}_{\perp}\right)=\frac{v\left[A\left(k_{\perp}+v\right) e^{v D}-B\left(k_{\perp}-v\right)\right]}{k_{\perp} C}, \\
& \phi^{-}\left(\mathbf{k}_{\perp}\right)=\left(v / k_{\perp} C\right) \times\left[-2 k_{\perp} B+\left[\left(k_{\perp}+v\right) e^{v D}+\left(k_{\perp}-v\right) e^{-v D}\right] A\right], \\
& \phi^{+}\left(\mathbf{k}_{\perp}\right)=\left(-v / k_{\perp} C\right)\left[-2 k_{\perp} A+\left[\left(k_{\perp}+v\right) e^{v D}+\left(k_{\perp}-v\right) e^{-v D}\right] B\right] \\
& C\left(k_{\perp}\right)=e^{-v D}\left(k_{\perp}-v\right)^{2}-e^{v D}\left(k_{\perp}+v\right)^{2}, v=\sqrt{k_{\perp}^{2}+\lambda_{a b}^{-2}}, \\
& A\left(\mathbf{k}_{\perp}\right)=\frac{1}{2 D} \sum_{m} g_{l}\left(\mathbf{k}_{\perp}, k_{m}\right) \nu_{z}^{v i}\left(\mathbf{k}_{\perp}, k_{m}\right), B\left(\mathbf{k}_{\perp}\right)=\frac{1}{2 D} \sum_{m} e^{-i k_{m} d} g_{l}\left(\mathbf{k}_{\perp}, k_{m}\right) \nu_{z}^{v i}\left(\mathbf{k}_{\perp}, k_{m}\right) \\
& g_{l}(k)=\frac{\phi_{0}}{1+k^{2} \lambda_{a b}^{2}} .
\end{aligned}
$$

The summation in the above expressions is carried out over $k_{z}=k_{m} \equiv m \pi / D, m=$ $0, \pm 1, \pm 2, \ldots$ and $\perp$ stands for the vector component parallel to the $x y$ plane. Following 
Ref. 29 we introduce here the Fourier transform $\nu^{v i}\left(\mathbf{k}_{\perp}, k_{m}\right)$ of the vorticity distribution $\nu^{v i}(\mathbf{r})$ :

$$
\begin{gathered}
\nu_{\perp}^{v i}\left(\mathbf{k}_{\perp}, k_{m}\right)=-2 i \int d^{2} r_{\perp} e^{-i \mathbf{k}_{\perp} \mathbf{r}_{\perp}} \int_{-D}^{0} d z \sin \left(k_{m} z\right) \nu_{\perp}^{v i}\left(\mathbf{r}_{\perp}, z\right) \\
\nu_{z}^{v i}\left(\mathbf{k}_{\perp}, k_{m}\right)=2 \int d^{2} r_{\perp} e^{-i \mathbf{k}_{\perp} \mathbf{r}_{\perp}} \int_{-D}^{0} d z \cos \left(k_{m} z\right) \nu_{z}^{v i}\left(\mathbf{r}_{\perp}, z\right) .
\end{gathered}
$$

For a pair of curved vortices shifted in the $y$ direction at a certain distance $R$ the expressions (B2) and (B3) take the form:

$$
\begin{array}{r}
\nu_{x}^{v i}\left(\mathbf{k}_{\perp}, k_{m}\right)=0, \\
\nu_{y}^{v i}\left(\mathbf{k}_{\perp}, k_{m}\right)=-2 i\left(1+e^{-i k_{y} R}\right) \int_{-D}^{0} y^{\prime}(z) e^{-i k_{y} y(z)} \sin \left(k_{m} z\right) d z, \\
\nu_{z}^{v i}\left(\mathbf{k}_{\perp}, k_{m}\right)=2\left(1+e^{-i k_{y} R}\right) \int_{-D}^{0} e^{-i k_{y} y(z)} \cos \left(k_{m} z\right) d z .
\end{array}
$$

To find the vortex-vortex interaction energy we should take the terms in Eq. (B1) which depend on mutual vortex arrangement:

$$
\varepsilon_{i n t}=\varepsilon_{0}\left(\varepsilon_{i n t}^{v i}+\varepsilon_{i n t}^{s t r a y}+\varepsilon_{i n t}^{v a c}\right)
$$

where $\varepsilon_{0}=\phi_{0}^{2} / 16 \pi^{3} \lambda_{a b}$ and

$$
\begin{aligned}
& \epsilon_{i n t}^{v i}=\pi \int_{0}^{\infty} \nu d \nu \int_{-\tilde{D}}^{0} d \zeta_{1} \int_{-\tilde{D}}^{0} d \zeta_{2}\left\{J_{0}\left[\nu\left(\eta\left(\zeta_{2}\right)-\eta\left(\zeta_{1}\right)+\tilde{R}\right)\right]+J_{0}\left[\nu\left(\eta\left(\zeta_{2}\right)-\eta\left(\zeta_{1}\right)-\tilde{R}\right)\right]\right\} \\
& \times\left(\Pi_{1}\left(\nu, \zeta_{1}, \zeta_{2}\right)+\Pi_{2}\left(\nu, \zeta_{1}, \zeta_{2}\right)\right), \\
& \epsilon_{\text {int }}^{\text {stray }}=4 \pi \int_{0}^{\infty} \nu d \nu \int_{-\tilde{D}}^{0} d \zeta_{1} \int_{-\tilde{D}}^{0} d \zeta_{2}\left\{J_{0}\left[\nu\left(\eta\left(\zeta_{2}\right)-\eta\left(\zeta_{1}\right)+\tilde{R}\right)\right]+J_{0}\left[\nu\left(\eta\left(\zeta_{2}\right)-\eta\left(\zeta_{1}\right)-\tilde{R}\right)\right]\right\} \\
& \times \frac{\Pi_{3}\left(\nu, \zeta_{1}, \zeta_{2}\right)}{\tau(\nu) \sinh [\tau(\nu) \tilde{D}]\left\{e^{-\tau(\nu) \tilde{D}}[\nu-\tau(\nu)]^{2}-e^{\tau(\nu) \tilde{D}}[\nu+\tau(\nu)]^{2}\right\}^{2}}, \\
& \epsilon_{\text {int }}^{v a c}=4 \pi \int_{0}^{\infty} \nu d \nu \int_{-\tilde{D}}^{0} d \zeta_{1} \int_{-\tilde{D}}^{0} d \zeta_{2}\left\{J_{0}\left[\nu\left(\eta\left(\zeta_{2}\right)-\eta\left(\zeta_{1}\right)+\tilde{R}\right)\right]+J_{0}\left[\nu\left(\eta\left(\zeta_{2}\right)-\eta\left(\zeta_{1}\right)-\tilde{R}\right)\right]\right\} \\
& \times \frac{\Pi_{4}\left(\nu, \zeta_{1}, \zeta_{2}\right)}{\nu \sinh ^{2}[\tau(\nu) \tilde{D}]\left\{e^{-\tau(\nu) \tilde{D}}[\nu-\tau(\nu)]^{2}-e^{\tau(\nu) \tilde{D}}[\nu+\tau(\nu)]^{2}\right\}^{2}},
\end{aligned}
$$




$$
\tau(\nu)=\sqrt{1+\Gamma^{2} \nu^{2}}, \quad u(\nu)=\sqrt{1+\nu^{2}}
$$

Here we have introduced dimensionless coordinates $\eta=y / \lambda_{a b}, \zeta=z / \lambda_{a b}$, dimensionless wave number $\nu=q \lambda_{a b}$, and use the notations

$$
\begin{aligned}
& \Pi_{1}\left(\nu, \zeta_{1}, \zeta_{2}\right)=\eta^{\prime}\left(\zeta_{1}\right) \eta^{\prime}\left(\zeta_{2}\right) \frac{\sinh \left[\tau(\nu)\left(\tilde{D}+\left(\zeta_{1}+\zeta_{2}-\left|\zeta_{1}-\zeta_{2}\right|\right) / 2\right)\right] \sinh \left[\tau(\nu)\left(\left(-\zeta_{1}-\zeta_{2}-\left|\zeta_{1}-\zeta_{2}\right|\right) / 2\right)\right]}{\tau(\nu) \sinh [\tilde{D} \tau(\nu)]} \\
& \Pi_{2}\left(\nu, \zeta_{1}, \zeta_{2}\right)=\frac{\tau(\nu) \cosh \left[\tau(\nu)\left(\tilde{D}+\left(\zeta_{1}+\zeta_{2}-\left|\zeta_{1}-\zeta_{2}\right|\right) / 2\right)\right] \cosh \left[\tau(\nu)\left(\left(-\zeta_{1}-\zeta_{2}-\left|\zeta_{1}-\zeta_{2}\right|\right) / 2\right)\right]}{\nu^{2} \sinh [\tilde{D} \tau(\nu)]} \\
& -\frac{\cosh \left[u(\nu)\left(\tilde{D}+\left(\zeta_{1}+\zeta_{2}-\left|\zeta_{1}-\zeta_{2}\right|\right) / 2\right)\right] \cosh \left[u(\nu)\left(\left(-\zeta_{1}-\zeta_{2}-\left|\zeta_{1}-\zeta_{2}\right|\right) / 2\right)\right]}{\nu^{2} u(\nu) \sinh [\tilde{D} u(\nu)]} \\
& \Pi_{3}\left(\nu, \zeta_{1}, \zeta_{2}\right)=\left\{\nu^{2} \cosh [\tau(\nu) \tilde{D}]+\tau(\nu)^{2} \cosh [\tau(\nu) \tilde{D}]+2 \nu \tau(\nu) \sinh [\tau(\nu) \tilde{D}]\right\} \\
& \times\left\{\cosh \left[\tau(\nu)\left(\tilde{D}+\zeta_{1}\right)\right] \cosh \left[\tau\left(\tilde{D}+\zeta_{2}\right)\right]+\cosh \left[\tau(\nu) \zeta_{1}\right] \cosh \left[\tau(\nu) \zeta_{1}\right]\right\} \\
& \left.+\cosh \left[\tau(\nu)\left(\tilde{D}+\zeta_{1}\right)\right]\right) \cosh \left[\tau(\nu) \zeta_{2}\right]+\cosh \left[\tau(\nu) z_{1}\right] \cosh \left[\tau(\nu)\left(\zeta_{2}+\tilde{D}\right)\right] \\
& \Pi_{4}\left(\nu, \zeta_{1}, \zeta_{2}\right)=\left\{2 \nu^{2} \cosh ^{2}[\tau(\nu) \tilde{D}]+\sinh ^{2}[\tau(\nu) \tilde{D}]+2 \nu \tau(\nu) \sinh [\tau(\nu) \tilde{D}] \cosh [\tau(\nu) \tilde{D}]\right\} \times \\
& \left\{\cosh \left[\tau(\nu)\left(\tilde{D}+\zeta_{1}\right)\right] \cosh \left[\tau(\nu)\left(\tilde{D}+\zeta_{2}\right)\right]+\cosh \left[\tau(\nu) \zeta_{1}\right] \cosh \left[\tau(\nu) \zeta_{1}\right]\right\}- \\
& 2\left\{\nu^{2} \cosh [\tau(\nu) \tilde{D}]+\nu \tau \sinh [\tau(\nu) \tilde{D}]\right\} \times \\
& \left\{\cosh \left[\tau(\nu)\left(\tilde{D}+\zeta_{1}\right)\right] \cosh \left[\tau(\nu) \zeta_{2}\right]+\cosh \left[\tau(\nu) \zeta_{1}\right] \cosh \left[\tau(\nu)\left(\zeta_{2}+\tilde{D}\right)\right]\right\} .
\end{aligned}
$$

The dimensionless thickness of the film $\tilde{D}$ and dimensionless intervortex distance $\tilde{R}$ are measured in the units of $\lambda_{a b}$.

1 A. A. Abrikosov, Fundamentals of the Theory of Metals (North-Holland, Amsterdam, 1988 ).

2 R. Joynt, Rev. Mod. Phys. 74, 235 (2002). 
3 A. S. Mel'nikov, Zh. Eksp. Teor. Fiz. 101, 1978 (1992) [JETP 74, 1059 (1992)].

4 M. Houzet, A. I. Buzdin, Europhys. Lett. 50, 375 (2000).

5 A. I. Buzdin, A. S. Mel'nikov, A. V. Samokhvalov, T. Akashi, T. Masui, T. Matsuda, S. Tajima, H. Tadatomo, and A. Tonomura, Phys. Rev. B 79, 094510 (2009).

6 A. I. Buzdin and A. Yu. Simonov, JETP Lett. 51, 191 (1990).

7 A. M. Grishin, A. Yu. Martynovich and S. V. Yampolskii, Sov. Phys. JETP 70, 1089 (1990) [Zh. Eksp. Teor. Fiz. 97, 1930 (1990)].

8 V. G. Kogan, N. Nakagawa and S. L. Thiemann, Phys. Rev. B 42, 2631 (1990).

9 S. J. Bending and M. J. W. Dodgson, J. Phys.: Cond. Matter 17, R955 (2005).

10 J. Pearl, Appl. Phys. Lett. 5, 65 (1964).

11 W. E. Lowrence and S. Doniach, in Proceedings of 12th International Conference on Low Temperature Physics, Kyoto 1970, edited by E.Kanda (Keigaku, Tokyo, 1970), p.361.

12 A. Buzdin, D. Feinberg, J. de Physique 51, 1971 (1990).

13 J. R. Clem, Phys. Rev. B 43, 7837 (1991).

14 E. H. Brandt, Phys. Rev. B, 48, 6699 (1993).

15 A. Yu. Martynovich, Sov. Phys. JETP 78, 489 (1994) [Zh. Eksp. Teor. Fiz. 105, 912 (1994)].

16 P. L. Gammel, D. J. Bishop, J. P. Rice and D. M. Ginsberg, Phys. Rev. Lett. 68, 3343 (1992).

17 H. F. Hess, C. A. Murray, and J. V. Waszczak, Phys. Rev. Lett. 69, 2138 (1992).

18 A. Grigorenko, S. Bending, T. Tamegai, S. Ooi, and M. Henini, Nature (London) 414, 728 (2001).

19 T. Matsuda, O. Kamimura, H. Kasai, K. Harada, T. Yoshida, T. Akashi, A. Tonomura, Y. Nakayama, J. Shimoyama, K. Kishio, T. Hanaguri, and K. Kitazawa, Science 294, 2136 (2001).

20 A. Tonomura, H. Kasai, O. Kamimura, T. Matsuda, K. Harada, T. Yoshida, T. Akashi, J. Shimoyama, K. Kishio, T. Hanaguri, K. Kitazawa, T. Masui, S. Tajima, N. Koshizuka, P. L. Gammel, D. Bishop, M. Sasase, and S. Okayasu, Phys. Rev. Lett. 88, 237001 (2002).

21 V. Pudikov, Physica C 212, 155 (1993).

22 T. Pe, M. Benkraouda, and J. R. Clem, Phys. Rev. B 55, 6636 (1997).

23 L. N. Bulaevskii, M. Ledvij, and V. G. Kogan, Phys. Rev. B 46, 366 (1992).

24 A. E. Koshelev, Phys. Rev. Lett. 83, 187 (1999).

25 L. N. Bulaevskii, M. Maley, H. Safar, and D. Dominguez, Phys. Rev. B 53, 6634 (1996).

26 A. Buzdin and I. Baladie, Phys. Rev. Lett. 88, 147002 (2002). 
27 R. A. Klemm and J. R. Clem, Phys. Rev. B, 21, 1868 (1980).

28 J. Pearl, J. Appl. Phys. 37, 4139 (1966).

29 G. Carneiro, E. H. Brandt, Phys. Rev. B 61, 6370 (2000).

30 A. I. Buzdin and A. Yu. Simonov, Sov. Phys. JETP 71, 1165 (1990) [Zh. Eksp. Teor. Fiz. 98, 2074 (1990)].

31 A. I. Buzdin and A. Yu. Simonov, Physica C 175, 143 (1991). 


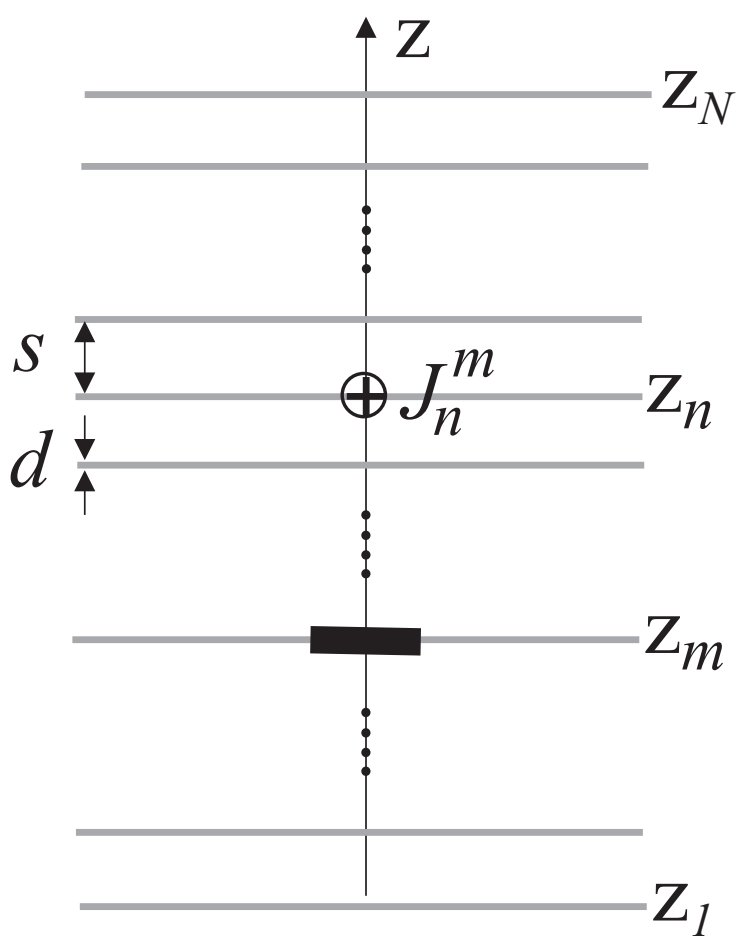

FIG. 1: A single $2 D$ pancake vortex positioned in the $m$-th layer of a finite layered structure, $d$ is a thickness of the superconducting layer, and $s$ is the distance between the layers. 

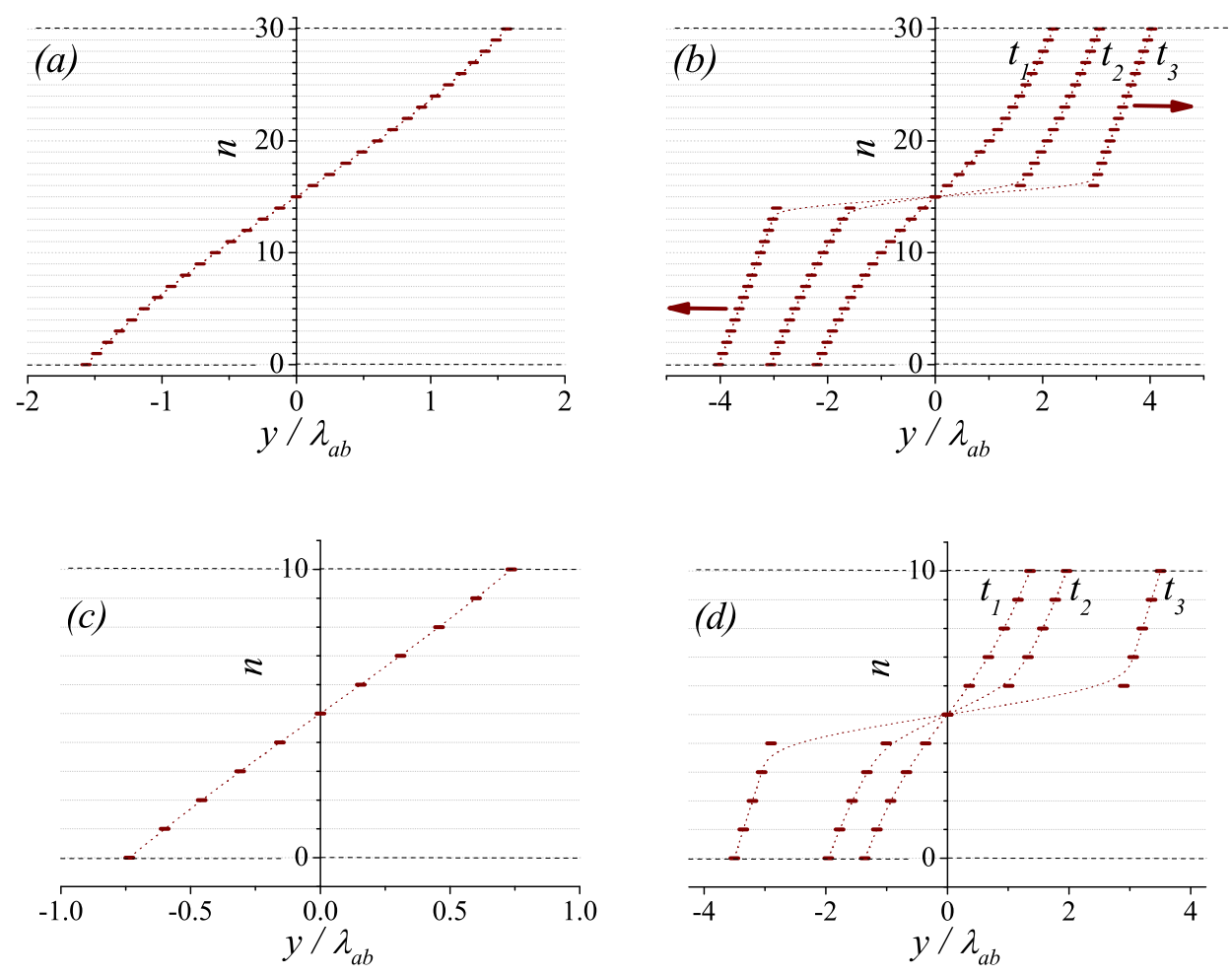

FIG. 2: (Color online) Configurations of $N=31$ (panels a, b) and $N=11$ (panels c, d) pancakes in a finite stack in the presence of the applied in-plane magnetic field $H_{a}$. (a) The force-balanced (equilibrium) configuration of pancakes for $H_{a}=0.2 H_{0}<H^{*}$. (b) Pancake configurations at sequential time points $t_{1}<t_{2}<t_{3}$ for $H_{a}=0.22 H_{0}>H^{*}$. For the structure with $N=31$ we find $H^{*} \simeq 0.21 H_{0}$. (c) The force-balanced (equilibrium) configuration of pancakes for $H_{a}=0.35 H_{0}<$ $H^{*}$. (d) Pancake configurations at sequential time points $t_{1}<t_{2}<t_{3}$ for $H_{a}=0.4 H_{0}>H^{*}$. For the structure with $N=11$ we find $H^{*} \simeq 0.38 H_{0}$. Here $H_{0}=\phi_{0} / 2 \pi \lambda_{a b}^{2}, \Lambda=10 \lambda_{a b}$, and $s=0.1 \lambda_{a b}$. 

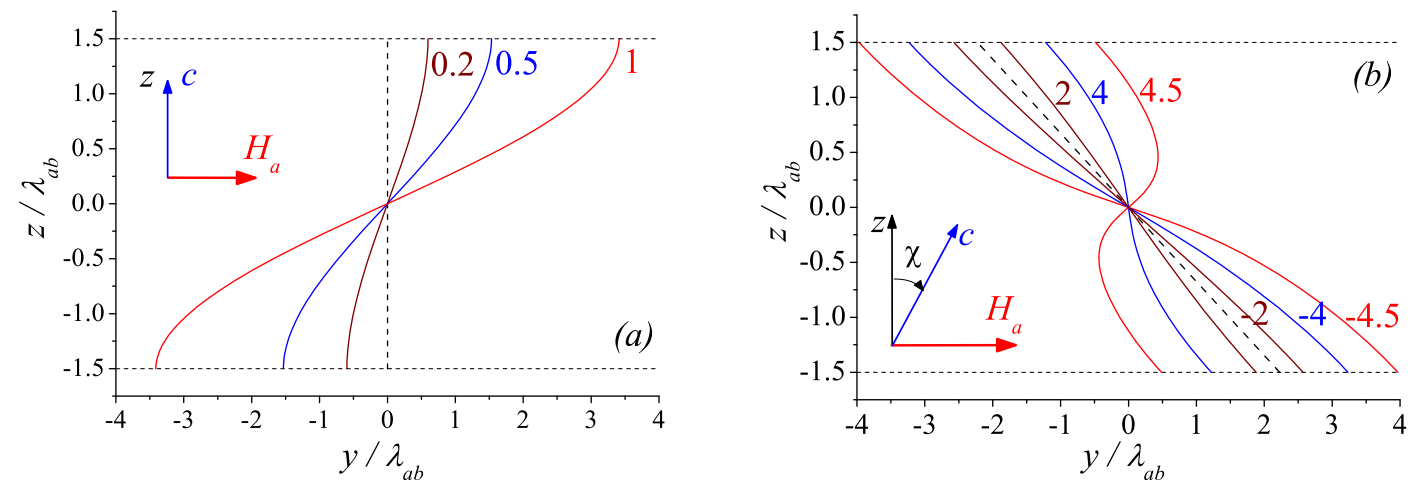

FIG. 3: (Color online) Typical configurations of the vortex lines in the film of the thickness $D=3 \lambda_{a b}$ for the anisotropy parameter $\Gamma=5$ and for different values of in-plane magnetic field $H_{\|}=H_{a} \mathbf{y}_{0}$. (a) The anisotropy axis is perpendicular to the film plane $\left(\chi=0^{o}, H_{c 1}^{(0)} \simeq 1.74 H_{a b}\right)$. (b) The anisotropy axis is tilted with respect to the $z$ axis $\left(\chi=30^{\circ}, H_{c 1}^{(\chi)} \simeq 4.6 H_{a b}\right)$ The numbers near the curves denote the values of the ratio $H_{a} / H_{a b}$. The dashed line shows the shape of a vortex line in the absence of the in-plane magnetic field.

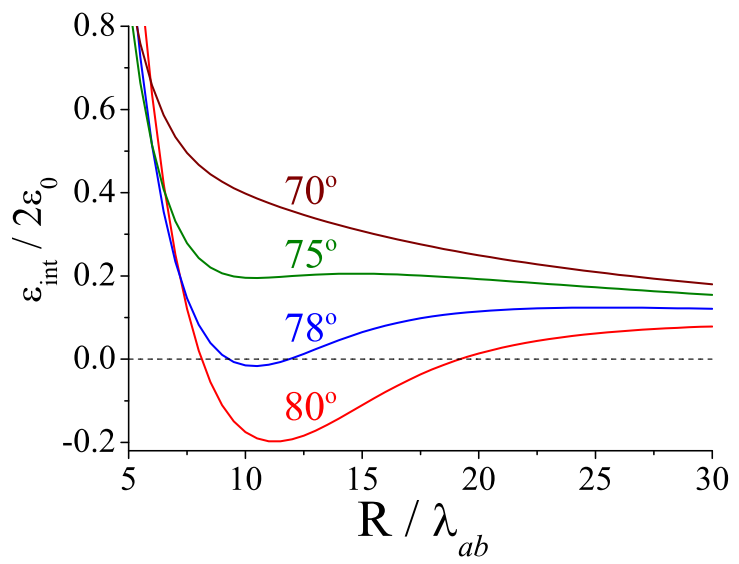

FIG. 4: (Color online) Typical plots of the interaction energy per vortex [Eqs. (33) and(134)] vs the distance $\mathrm{R}$ between two vortices for a film of thickness $d=3 \lambda_{a b}$ and different tilting angles $\gamma=70^{\circ}, 75^{\circ}, 78^{\circ}, 80^{\circ}\left(\varepsilon_{0}=\phi_{0}^{2} / 16 \pi^{3} \lambda_{a b}\right)$. 

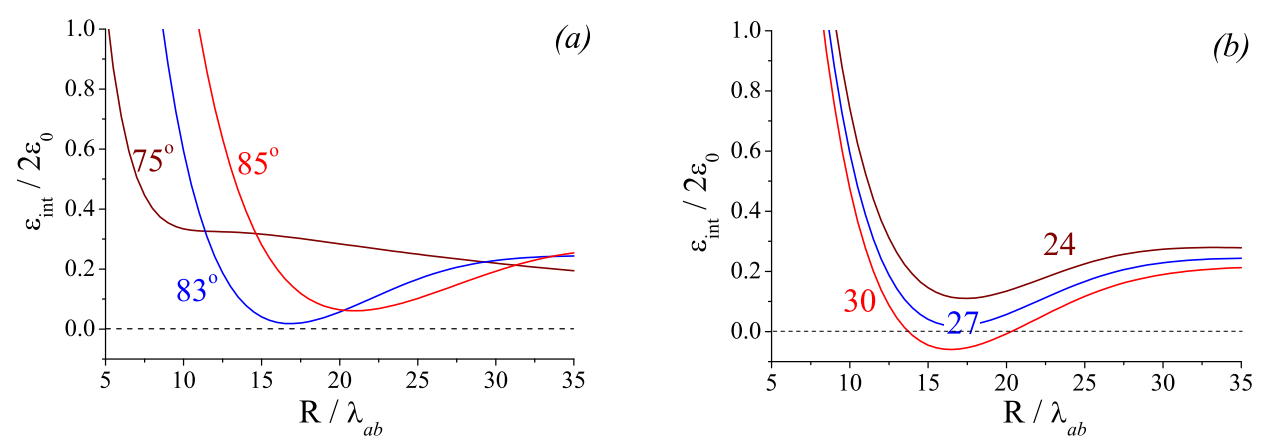

FIG. 5: (Color online) Typical plots of the interaction energy per vortex [Eqs. (38), (39)] vs the distance $\mathrm{R}$ between two tilted vortices for an anisotropic film of the thickness $D=3 \lambda_{a b}$. (a) Interaction energy for the anisotropy parameter $\Gamma=27$ and different tilting angles. The numbers near the curves denote the values of tilting angle $\gamma$. (b) Interaction energy for $\gamma=83^{\circ}$ and different values of anisotropy parameter. The numbers near the curves denote the values of $\Gamma$.
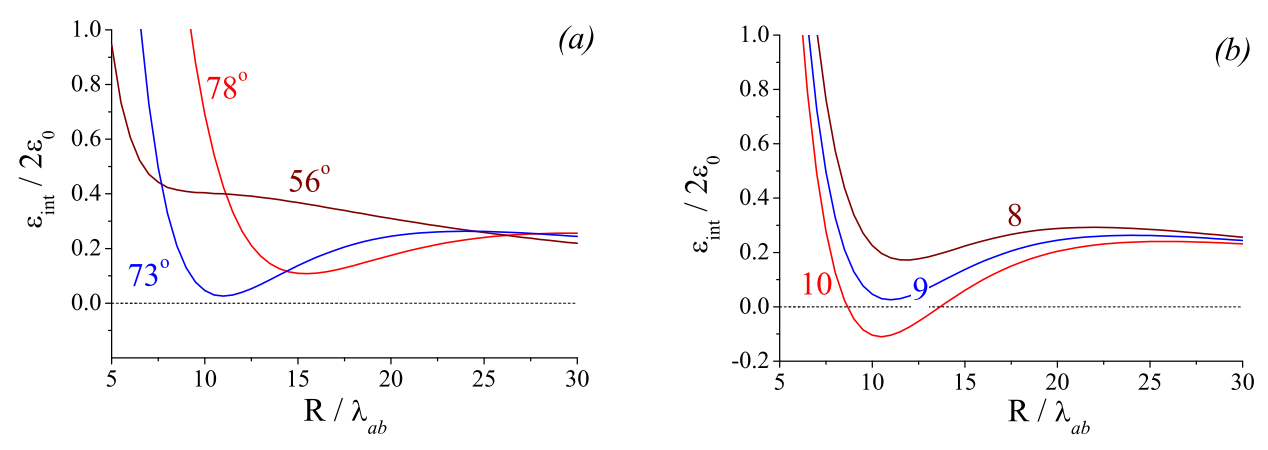

FIG. 6: (Color online) Typical plots of the interaction energy per vortex [Eqs. (38), (39)] $]$ vs the distance $\mathrm{R}$ between two tilted vortices for an anisotropic film of the thickness $D=10 \lambda_{a b}$. (a) Interaction energy for the anisotropy parameter $\Gamma=9$ and different tilting angles. The numbers near the curves denote the values of tilting angle $\gamma$. (b) Interaction energy for $\gamma=73^{\circ}$ and different values of anisotropy parameter. The numbers near the curves denote the values of $\Gamma$. 

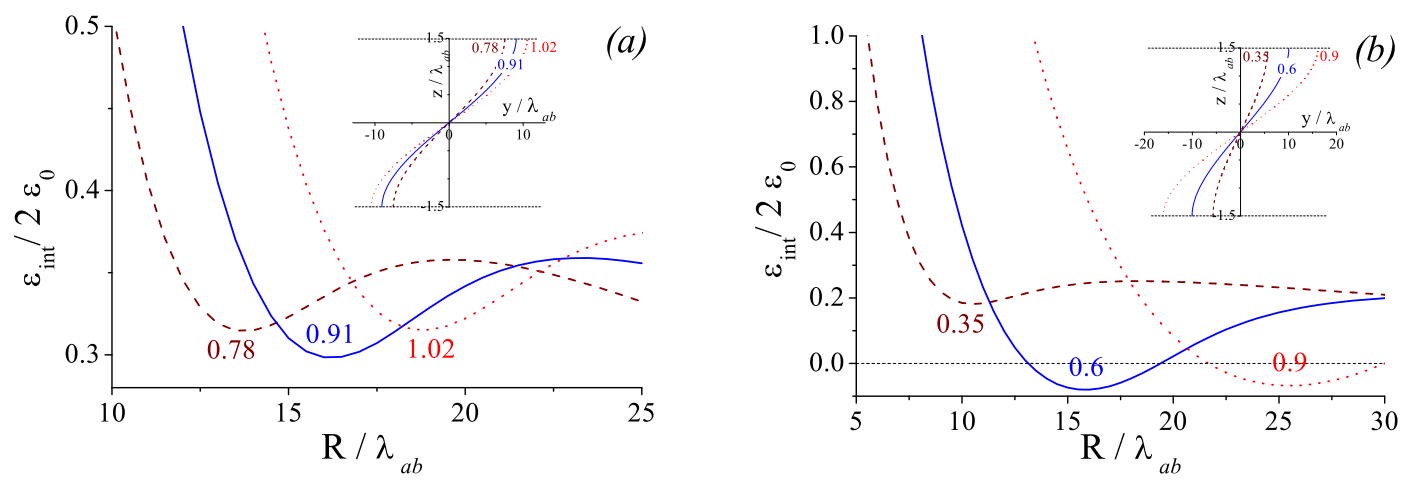

FIG. 7: (Color online) Typical plots of the interaction energy per vortex (37) vs the distance $R$ between two curved vortices for an anisotropic film of the thickness $D=3 \lambda_{a b}$ : (a) $\Gamma=15$; (b) $\Gamma=27$. The numbers near the curves denote the values of the ratio $H_{a} / H_{a b}$. The shape of vortex lines is schematically shown in the insets.
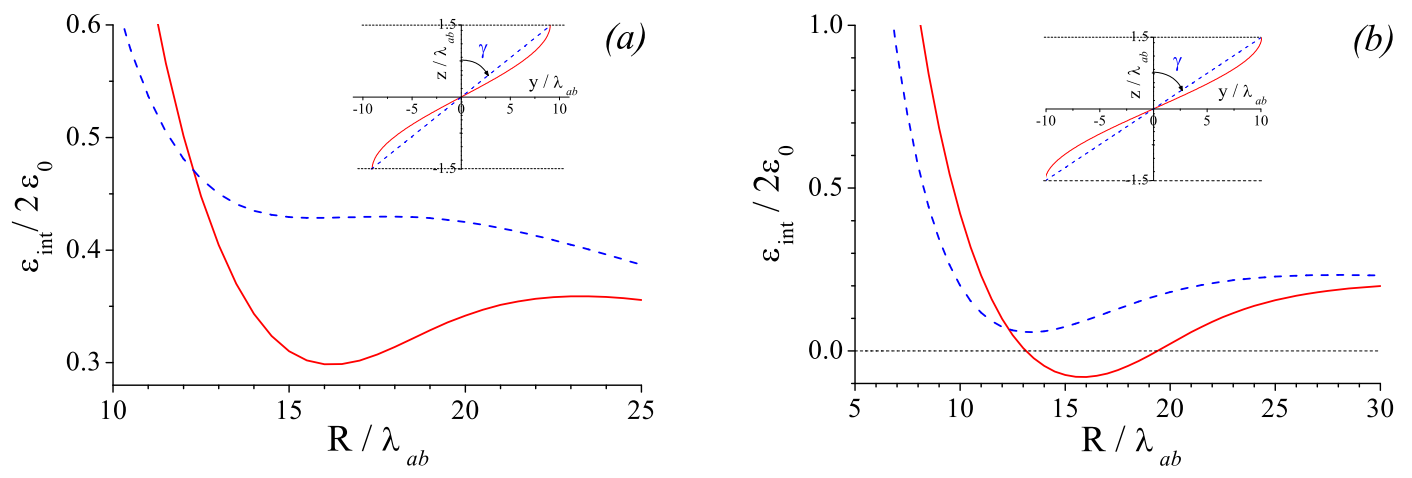

FIG. 8: (Color online) Comparison of the vortex-vortex interaction potentials for curved [Eq. (37)] (solid lines) and straight tilted [Eqs. (38), (39)] (dashed lines) vortices for an anisotropic film of the thickness $D=3 \lambda_{a b}$ with different anisotropy parameters: (a) $\Gamma=15, H_{a}=0.91 H_{a b}\left(\gamma=80.6^{\circ}\right)$; (b) $\Gamma=27, H_{a}=0.6 H_{a b}\left(\gamma=81.5^{\circ}\right)$. The shape of vortex lines is schematically shown in the insets. 

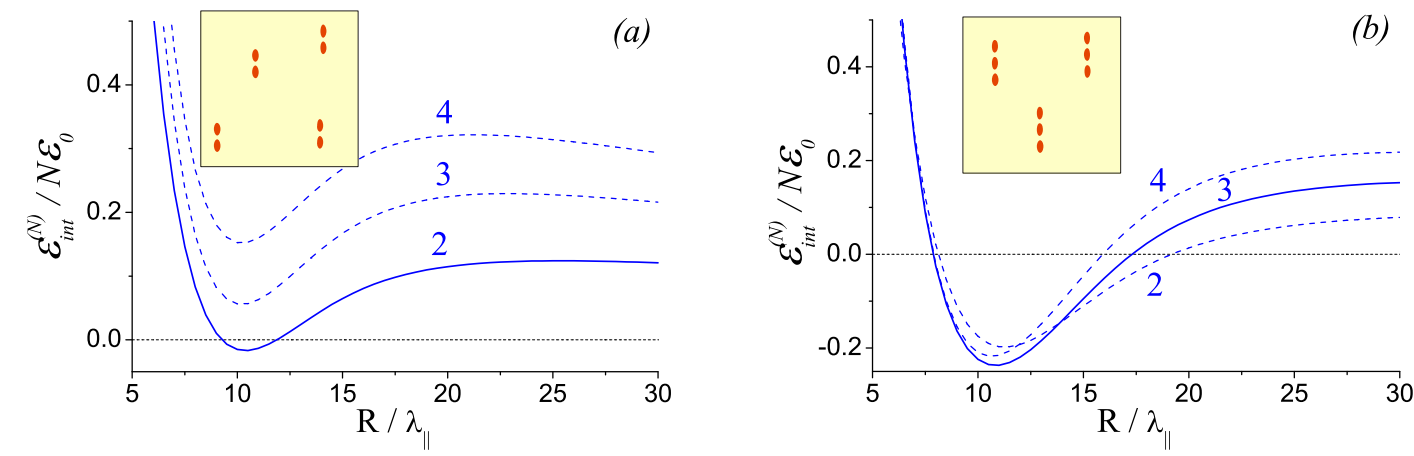

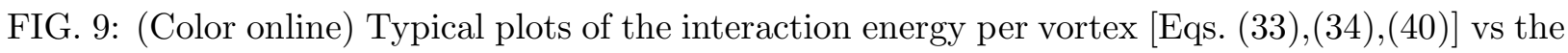
intervortex distance $R$ in an equidistant chain of $\mathrm{N}$ vortices in a stack of decoupled superconducting layers $\left(D=3 \lambda_{a b}\right)$ : (a) $\gamma=78^{\circ}$; (b) $\gamma=80^{\circ}$. The numbers near the curves denote the number $N$ of vortices in molecule. Inserts show schematic pictures of vortex matter consisting of dimeric (a) and trimeric (b) molecules.

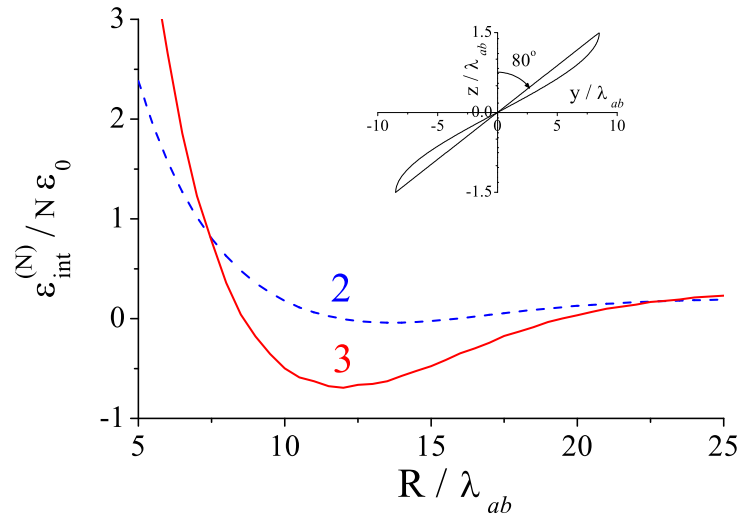

FIG. 10: (Color online) Typical plots of the interaction energy per vortex vs the intervortex distance $\mathrm{R}$ in an equidistant chain of $\mathrm{N}$ vortices for $d=3 \lambda_{a b}, \Gamma=27, H_{a}=0.513 H_{a b}\left(\gamma=80^{\circ}\right)$. The numbers near the curves denote the number $\mathrm{N}$ of vortices in a molecule. The shape of vortex line and effective tilting angle $\gamma$ are schematically shown in the inset. 

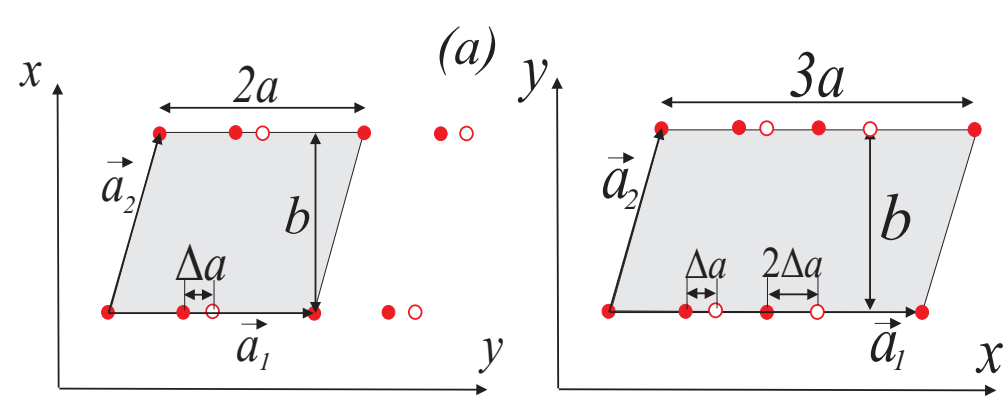

(b)

FIG. 11: (Color online) Vortex lattice with two $M=2(a)$ and three $M=3(b)$ vortices per a primitive cell.
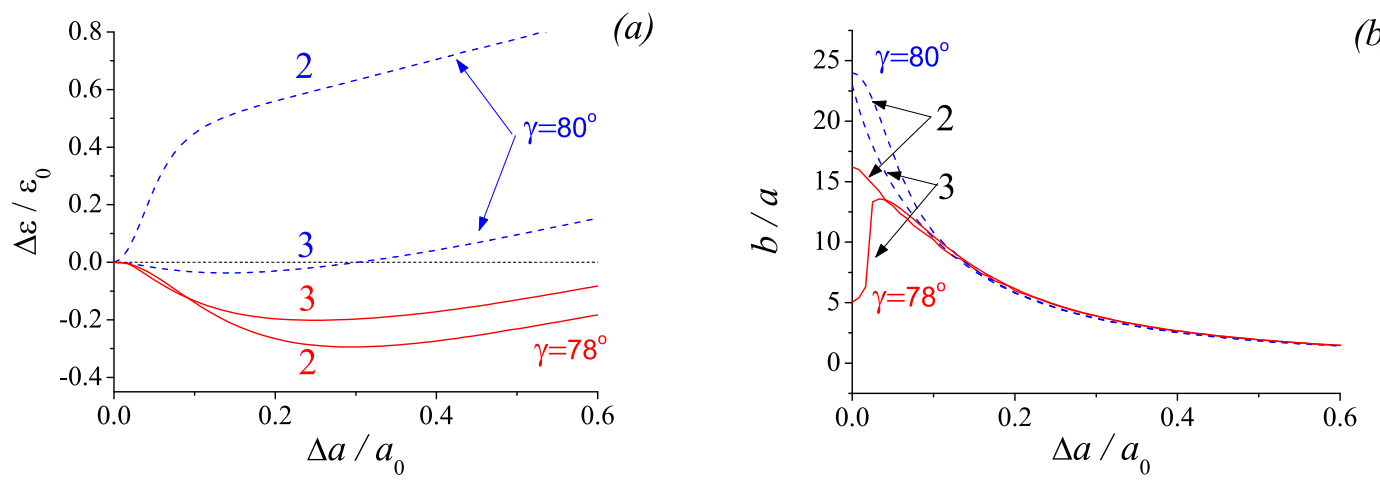

FIG. 12: (Color online)(a) The energy difference $\Delta \varepsilon_{c}$ vs the relative displacement $\Delta a$ of vortex sublattices for different tilting angles $\gamma=78^{\circ}$ (solid line) and $\gamma=80^{\circ}$ (dashed line) and different number of flux quanta per unit cell $M=2$, 3. (b) Lattice deformation ratio $\sigma=b / a$ vs the relative displacement $\Delta a$ of vortex sublattices for different tilting angles $\gamma=78^{\circ}$ (solid line) and $\gamma=80^{\circ}$ (dashed line) and different number of flux quanta per unit cell $M=2,3$. Here we put $a_{0}=60 \lambda_{a b}$. The numbers near the curves denote the number $M$ of vortices per unit cell. 